\title{
A TV BASED RESTORATION MODEL WITH LOCAL CONSTRAINTS
}

By
A. Almansa
C. Ballester
V. Caselles
and
G. Haro

\section{IMA Preprint Series \# 2119}

(May 2006)

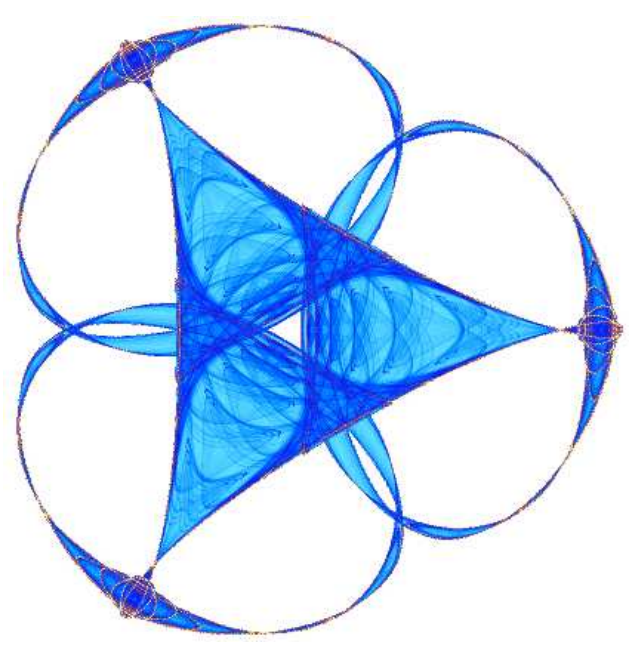

INSTITUTE FOR MATHEMATICS AND ITS APPLICATIONS

UNIVERSITY OF MINNESOTA 400 Lind Hall 207 Church Street S.E. Minneapolis, Minnesota 55455-0436

Phone: 612-624-6066 Fax: 612-626-7370

URL: http://www.ima.umn.edu 


\title{
A TV based restoration model with local constraints
}

\author{
A. Almansa * \\ C. Ballester ${ }^{\dagger}$ \\ V. Caselles $\ddagger$ \\ G. Haro ${ }^{\S}$
}

May 3, 2006

\begin{abstract}
We propose in this paper a total variation based restoration model which incorporates the image acquisition model $z=h * u+n$ ( $h$ denotes the blurring kernel and $n$ a white Gaussian noise) as a set of local constraints. These constraints, one for each pixel of the image, express the fact that the variance of the noise can be estimated from the residuals $h * u-z$ if we use a neighborhood of each pixel. This is motivated by the fact that the usual inclusion of the image acquisition model as a single constraint expressing a bound for the variance of the noise does not give satisfactory results if we wish to simultaneously recover textured regions and obtain a good denoising of the image. We use Uzawa's algorithm to minimize the total variation subject to the proposed family of local constraints and we display some experiments using this model.
\end{abstract}

Keywords: Image restoration, Total Variation, variational methods, satellite images.

AMS (MOS) subject classification: 68U10, 65K10, 65J20, 94A08

Suggested running head: A TV based restoration model with local constraints

${ }^{*}$ InCo, Facultad de Ingeniería, Universidad de la República, Montevideo, Uruguay almansa@fing.edu.uy

${ }^{\dagger}$ Dept. Tecnologia, Universitat Pompeu Fabra, Passeig de Circumvalació, 8, 08003 Barcelona, Spain, coloma.ballester@upf.edu

${ }^{\ddagger}$ Dept. Tecnologia, Universitat Pompeu Fabra, Passeig de Circumvalació, 8, 08003 Barcelona, Spain, vicent.caselles@upf.edu

${ }^{\S}$ Institute for Mathematics and its Applications, University of Minnesota, 400 Lind Hall, 207 Church Street S.E. Minneapolis, MN 55455, USA, haro@ima.umn.edu 


\section{Introduction}

We assume that the image acquisition system may be modelled by the following image formation model

$$
z(i, j)=h * u(i . j)+n(i, j), \quad(i, j) \in\{1, \ldots, N\}^{2}
$$

where $u: \mathbb{R}^{2} \rightarrow \mathbb{R}$ denotes the ideal undistorted image, $h: \mathbb{R}^{2} \rightarrow \mathbb{R}$ is a blurring kernel, $z$ is the observed sampled image which is represented as a function $z:\{1, \ldots, N\}^{2} \rightarrow \mathbb{R}$, and $n(i, j)$ is, as usual, a white Gaussian noise with zero mean and standard deviation $\sigma$.

Let us denote by $\Omega_{N}$ the interval $\left.] 0, N\right]^{2}$. As in most of works, in order to simplify this problem, we assume that the functions $h$ and $u$ are periodic of period $N$ in each direction. That amounts to neglecting some boundary effects. Therefore, we assume that $h, u$ are functions defined in $\Omega_{N}$. To fix ideas, we assume that $h, u \in L^{2}\left(\Omega_{N}\right)$, so that $h * u$ is a continuous function in $\Omega_{N}$ [18] (which may be extended to a continuous periodic function in $\left.\mathbb{R}^{2}\right)$ and the samples $h * u(i, j),(i, j) \in\{1, \ldots, N\}^{2}$, have sense.

Our problem is to recover as much as possible of $u$, from our knowledge of the blurring kernel $h$, the statistics of the noise $n$, and the irregular samples $z$.

The problem of recovering $u$ from $z$ is ill-posed due to the ill-conditioning of the operator $H u=h * u$. Several methods have been proposed to recover $u$. Most of them can be classified as regularization methods which may take into account statistical properties (Wiener filters), information theoretic properties [13], a priori geometric models [31] or the functional analytic behavior of the image given in terms of its wavelet coefficients [14].

The typical strategy to solve this ill-conditioning is regularization. Probably one of the first examples of regularization method [33] consists in choosing between all possible solutions of (1) the one which minimized the Sobolev (semi) norm of $u$

$$
\int_{\Omega_{N}}|D u|^{2} d x
$$

Usually, the only information we know about the noise is statistical and limited to an estimate of its mean and its variance. In that case, the model equation (1) is incorporated as a set of constraints for $(2)$ :

$$
\begin{gathered}
\sum_{i, j=1}^{N} h * u(i, j)=\sum_{i, j=1}^{N} z(i, j), \\
\sum_{i, j=1}^{N}|h * u(i, j)-z(i, j)|^{2} \leq \sigma^{2} N^{2} .
\end{gathered}
$$

The first constraint corresponds to the assumption that the noise has zero mean, and the second amounts to say that $\sigma$ is an upper bound of the standard deviation of $n$. As it is observed in [11], assuming that $h * 1=1$ (energy preservation), it suffices to minimize (2) under the constraint (4), since the constraint (3) is automatically satisfied [11]. Thus, in practice the above problem is solved via the following unconstrained minimization problem

$$
\min _{u} \max _{\lambda \geq 0} \int_{\Omega_{N}}|D u|^{2} d x+\frac{\lambda}{2}\left[\frac{1}{N^{2}} \sum_{i, j=1}^{N}|h * u(i, j)-z(i, j)|^{2}-\sigma^{2}\right],
$$

where $\lambda \geq 0$ is a Lagrange multiplier. 
This formulation was an important step, but the results were not satisfactory, mainly due to the unability of the previous functional to resolve discontinuities (edges) and oscillatory textured patterns. The smoothness required by the Dirichlet integral (2) is too restrictive and information corresponding to high frequencies of $z$ is attenuated by it. Indeed, functions in $W^{1,2}(\Omega)$ (i.e., functions $u \in L^{2}(\Omega)$ such that $D u \in L^{2}(\Omega)$ ) cannot have discontinuities along rectifiable curves. These observations motivated the introduction of Total Variation in image restoration problems by L. Rudin, S. Osher and E. Fatemi in their work [31]. The a priori hypothesis is that functions of bounded variation (the $B V$ model) ([4]) are a reasonable functional model for many problems in image processing, in particular, for restoration problems ([31]). Typically, functions of bounded variation have discontinuities along rectifiable curves, being continuous in some sense (in the measure theoretic sense) away from discontinuities. The discontinuities could be identified with edges. The ability of total variation regularization to recover edges is one of the main features which advocates for the use of this model (its ability to describe textures is less clear, some textures can be recovered, but up to a certain scale of oscillation).

On the basis of the $B V$ model, Rudin-Osher-Fatemi [31] proposed to solve the following constrained minimization problem

$$
\begin{aligned}
& \text { Minimize } \int_{\Omega}|D u| d x \\
& \sum_{i, j=1}^{N}|h * u(i, j)-z(i, j)|^{2} \leq \sigma^{2} N^{2} .
\end{aligned}
$$

Notice that the image acquisition model (1) is only incorporated through a global constraint. Notice also that, assuming that $h * 1=1$ (energy preservation), the constraint (3) is automatically satisfied [11].

In practice, the above problem is solved via the following unconstrained formulation

$$
\min _{u} \max _{\lambda \geq 0} \int_{\Omega}|D u| d x+\frac{\lambda}{2}\left[\frac{1}{N^{2}} \sum_{i, j=1}^{N}|h * u(i, j)-z(i, j)|^{2}-\sigma^{2}\right]
$$

where $\lambda \geq 0$ is a Lagrange multiplier. The appropriate value of $\lambda$ can be computed using Uzawa's algorithm [16] so that the constraint in (4) is satisfied. Recall that if we interpret $\lambda^{-1}$ as a penalization parameter which controls the importance of the regularization term, and we set this parameter to be large, then homogeneous zones are well denoised while highly textured regions will loose a great part of its structure. On the contrary, if $\lambda^{-1}$ is set to be small, texture will be kept but noise will remain in homogeneous regions. On the other hand, as the authors of [8] observed, if we use the constrained formulation (6) or, equivalently (7), then the Lagrange multiplier does not produce satisfactory results since we do not keep textures and denoise flat regions simultaneously, and they proposed to incorporate the image acquisition model as a set of local constraints, adapted to the different regions of the image. Let us mention that the use of local constraints on regions of the image was initially proposed in [30].

Following [8], let $\left\{O_{1}, \ldots, O_{r}\right\}$ be a partition of $\{1, \ldots, N\}^{2}$ into regions, and let us denote

$$
I_{O_{l}}(u):=\frac{1}{\left|O_{l}\right|} \sum_{(i, j) \in O_{l}}|h * u(i, j)-z(i, j)|^{2}
$$

where $\left|O_{l}\right|$ denotes the number of pixels in $O_{l}$. In [8], the authors proposed to incorporate the image 
acquisition model (1) as a set of local constraints, and to replace (6) by the problem

$$
\begin{aligned}
& \min _{u} \int_{\Omega_{N}}|D u| \\
& \text { subject to } I_{O_{l}}(u) \leq \sigma^{2}, \quad \forall l=1, \ldots, r,
\end{aligned}
$$

whose unconstrained formulation can be written as

$$
\min _{u} \max _{\lambda_{l} \geq 0} \int_{\Omega_{N}}|D u|+\frac{1}{2} \sum_{l=1}^{r} \lambda_{l}\left(I_{O_{l}}(u)-\sigma^{2}\right),
$$

where $\lambda_{l}, l=1, \ldots, r$ is a set of Lagrange multipliers associated to the constraints in (9) (and can be solved with Uzawa's method). Let us recall that the partition $\left\{O_{l}\right\}_{l=1}^{r}$ can be obtained with the simplified version [22] of the Mumford-Shah segmentation algorithm [27] applied to the image $z$. The segmentation algorithm is stopped to ensure that regions $O_{l}$ have a sufficiently large area to ensure that the sum in (8) gives a good estimate of $\sigma^{2}[8]$.

Our purpose in this paper is to further develop this idea and to get rid of the segmentation of $\{1, \ldots, N\}^{2}$ involved in this process. For that we propose to replace the family of constraints in (9) by

$$
G *(h * u-z)(i, j) \leq \sigma^{2}, \quad \forall(i, j) \in\{1, \ldots, N\},
$$

where $G$ is a discrete convolution kernel such that $G(i, j)>0$ for all $(i, j) \in\{1, \ldots, N\}$. The effective support of $G$ must permit the estimate of the variance of the noise with (11). Then we minimize the total variation (or the functional $\int_{\Omega_{N}} \sqrt{\beta^{2}+|D u|^{2}}, \beta>0$ ) subject to the family of constraints (11), plus eventually the constraint (3). In this paper we study the well-posedness of this problem and show that it can be solved with Uzawa's method [16].

Many numerical algorithms have been proposed to minimize total variation (or similar models) subject to the constraint given in $(6)[31,19,1,6,35,11,12,15,10]$. Besides the work [8], let us mention two of them related to our work. In [23] the authors combined total variation minimization with a set of constraints of type $|\langle h * u-z, \psi\rangle| \leq \tau$ where $\psi$ varies along an orthonormal basis of wavelets (or a family of them) and $\tau>0$. The aim was also to construct an algorithm which preserves textures and has good denoising properties. As we will do here, these constraints were incorporated using Uzawa's algorithm. With the same spirit of denoising while preserving textures, in [20], the authors proposed to minimize total variation subject to a family of local constraints which control the local variance of the oscillatory part of the signal. The constraints are introduced via Lagrange multipliers with an approach similar to the one used in [31]. This amounts to adding a spatially varying fidelity term that locally controls the extent of denoising over image regions depending on their content. Besides the fact that we use Uzawa's algorithm and we try to address the problem of deconvolution and denoising, the work [20] is quite similar to our approach. A different, though related, approach was taken in [32] (see also [25]) where the regularization parameter is scale and space adaptive. In that case the local parameter $\lambda(x, y)$ is on the regularization term, i.e. $\int_{\Omega} \lambda(x, y)|D u|$. For edge dependent adaptive restoration, $\lambda(x, y)$ is chosen in such a way that it is inversely proportional to $|D \hat{u}|$, where $\hat{u}$ is a previous estimation of the restored image $u$ obtained using total variation restoration [32]. Weighted total variation was also used in [25] with the purpose of controlling ringing effects in image restoration.

Let us finally say that we assume that the variance of the noise $\sigma^{2}$ is known. Otherwise, one could use any of the different approaches to obtain accurate estimates of $\sigma^{2}$, in particular, those in $[5,17]$. The paper [17] also provides an analysis of the relation between $\sigma^{2}$ and the regularization parameter 
for linear restoration methods. The paper [21] proposes a set of local constraints on regions of the image depending on the local variance of the region. The paper [26] proposes a Bayesian framework for parameter estimation in restoration problems addressed from a Bayesian point of view.

Let us describe the plan of the paper. Section 2 contains some preliminaries on probability in order to estimate the size of the kernel $G$ needed to be able to estimate the variance of the noise from the residuals $h * u-z$ and correspondingly define the local constraints (11). In Section 3 we study the wellposedness of minimizing total variation (and some related functionals) under the family of constraints (11), and we propose a minimization algorithm based on Uzawa's method. Our study will be conducted in a discrete setting. Section 4 is devoted to the description of the data and the numerical experiments performed using the proposed model. We end up in Section 5 with some conclusions.

\section{Averaging reduces noise variance}

If we consider, as we will do it in the next section, local estimates of the variance of the noise $n$, we can be sure that our numerical implementation will approximate $\sigma^{2}$ with an error that is inversely proportional to the square root of the area of the region considered. In other words, we can be sure that if the region is big enough our estimate of the noise will be good enough.

Let us consider a discrete image model and write

$$
z(i, j)=h * u(i, j)+n(i, j), \quad(i, j) \in\{1, \ldots, N\}^{2},
$$

as described in (1) where $u$ denotes the ideal undistorted image, $h$ is a blurring kernel, $z$ is the observed sampled image which is represented as a function $z:\{1, \ldots, N\}^{2} \rightarrow \mathbb{R}$, and $n(i, j) \in N\left(0, \sigma^{2}\right)$ is a white Gaussian noise with zero mean and variance $\sigma^{2}$. Let $I$ be a subset of indexes in $\mathbb{N} \times \mathbb{I}, a_{i j} \geq 0$, $\sum_{(i, j) \in I} a_{i j}=1$.

$$
S_{I}=\sum_{(i, j) \in I} a_{i j}(z(i, j)-h * u(i, j))^{2}=\sum_{(i, j) \in I} a_{i j} n(i, j)^{2} .
$$

Then

$$
E\left(S_{I}\right)=\sigma^{2} \quad \text { and } \quad \operatorname{var}\left(S_{I}\right)=2 \sigma^{4} \sum_{(i, j) \in I} a_{i j}^{2} .
$$

By the Central Limit Theorem [28], we have

$$
P\left(\left|S_{I}-\sigma^{2}\right|>\sqrt{2} x \sigma^{2}\left(\sum_{(i, j) \in I} a_{i j}^{2}\right)^{1 / 2}\right) \rightarrow \frac{1}{\sqrt{2 \pi}} \int_{|t|>x} e^{-t^{2} / 2} d t .
$$

In other words, the expression

$$
\sum_{(i, j) \in I} a_{i j} n(i, j)^{2}
$$

represents $\sigma^{2}$ with an error proportional to $\left(\sum_{(i, j) \in I} a_{i j}^{2}\right)^{1 / 2}$. In particular, if $a_{i j}=\frac{1}{|I|}$, then $S_{I}$ represents $\sigma^{2}$ with an error proportional to $\frac{1}{\sqrt{|I|}}$. This has only a heuristic value, since it does not give a precise estimate on the approximation error.

Remark 1 If $a_{i j}$ are obtained by discretizing a 2D Gaussian variable of standard deviation $t$, i.e., if $G_{t}(x, y)=\frac{1}{2 \pi t^{2}} e^{-\left(x^{2}+y^{2}\right) / 2 t^{2}}$, then we may estimate $\sum_{(i, j) \in I} a_{i j}^{2}$ by $\int_{\mathbb{R}} \int_{\mathbb{R}} G_{t}(x, y)^{2} d x d y=\frac{1}{4 \pi t^{2}}$. 
To get an idea of the size of the neighborhood where we have to average the noise, let us consider the case $a_{i j}=\frac{1}{|I|}$. In this case

$$
S_{I}=\frac{1}{|I|} \sum_{(i, j) \in I} n(i, j)^{2}
$$

and, given $\alpha \in[0,1]$, we may write

$$
P\left(\left|S_{I}-\sigma^{2}\right|>\alpha \sigma^{2}\right)=P\left(\left|\sum_{(i, j) \in I}\left(\frac{n(i, j)}{\sigma}\right)^{2}-\right| I||>|I| \alpha\right) .
$$

Since $Z:=\sum_{(i, j) \in I}\left(\frac{n(i, j)}{\sigma}\right)^{2}$ is a $\chi^{2}(|I|)$, and for $|I|>30, \sqrt{2 \chi^{2}(|I|)}-\sqrt{2|I|-1}$ is a good approximation to the normal distribution [28], then the probability in (13) can be estimated by

$$
e:=1-\mathcal{N}(\sqrt{2|I|(1+\alpha)}-\sqrt{2|I|-1})+\mathcal{N}(\sqrt{2|I|(1-\alpha)}-\sqrt{2|I|-1}) .
$$

where we denote

$$
\mathcal{N}(x):=\frac{1}{\sqrt{2 \pi}} \int_{-\infty}^{x} e^{-y^{2} / 2} d y
$$

If $\alpha=0.1$, and $I$ is a disk of radius 13 (resp. 14), then $\sqrt{2|I|(1+\alpha)}-\sqrt{2|I|-1} \approx 1.606$ (resp. 1.727), $\sqrt{2|I|(1-\alpha)}-\sqrt{2|I|-1} \approx-1.657$ (resp. -1.786 ) and $e \approx 0.1031$ (res. 0.0854). This only provides a good estimate of the noise power when $\sigma^{2}$ is small, which is reasonable for satellite image restoration. When $\sigma^{2}$ is large then the neighborhood becomes large and we have no advantage in using this local method. For instance, if $\sigma=20$, if we want to estimate the noise power $\sigma^{2}=400$ within an interval with $\alpha=0.05$ (thus $\alpha \sigma^{2}=20$ ) and we take a disk of radius 20, then $\sqrt{2|I|(1+\alpha)}-$ $\sqrt{2|I|-1} \approx 1.2480, \sqrt{2|I|(1-\alpha)}-\sqrt{2|I|-1} \approx-1.2594$ and $e \approx 0.21$. If $I$ is a disk of radius 30, then $\sqrt{2|I|(1+\alpha)}-\sqrt{2|I|-1} \approx 1.8637, \sqrt{2|I|(1-\alpha)}-\sqrt{2|I|-1} \approx-1.8974$ and $e \approx 0.06$. Thus, at this level of approximation we need a disk of radius 30 and this may be too large for the structures contained in the image. Finally, let us mention that in practice we have used a gaussian window $G_{t}$ with variance $t^{2}$. Since $\int_{\mathbb{R}} \int_{\mathbb{R}} G_{t}(x, y)^{2} d x d y=\frac{1}{4 \pi t^{2}}$ we can think that it is equivalent to a disk of radius $R=2 t$.

\section{A restoration model with pointwise local constraints}

Our next purpose is to introduce a restoration model with local constraints and to explain the numerical approach to solve it. For that, let us introduce some notation. We denote by $X$ the Euclidean space $\mathbb{R}^{N \times N}$. The Euclidean scalar product and the norm in $X$ will be denoted by $\langle\cdot, \cdot\rangle_{X}$ and $\|\cdot\|_{X}$, respectively. Then the image $u \in X$ is the vector $u=(u(i, j))_{i, j=1}^{N}$, and the vector field $\xi$ is the map $\xi:\{1, \ldots, N\} \times\{1, \ldots, N\} \rightarrow \mathbb{R}^{2}$. If $u \in X$, the discrete gradient is a vector in $Y=X \times X$ given by

$$
\nabla^{+,+} u:=\left(\nabla_{x}^{+} u, \nabla_{y}^{+} u\right)
$$

where

$$
\begin{aligned}
& \nabla_{x}^{+} u(i, j)=\left\{\begin{array}{ccc}
u(i+1, j)-u(i, j) & \text { if } & i<N \\
0 & \text { if } & i=N
\end{array}\right. \\
& \nabla_{y}^{+} u(i, j)=\left\{\begin{array}{ccc}
u(i, j+1)-u(i, j) & \text { if } & j<N \\
0 & \text { if } & j=N
\end{array}\right.
\end{aligned}
$$


for $i, j=1, \ldots, N$. Other choices of the gradient are possible, this one will be convenient for the developments below. We denote the euclidean norm of a vector $v \in \mathbb{R}^{2}$ by $|v|$.

Let us define the discrete functional

$$
J_{d}^{\beta}(u)=\sum_{1 \leq i, j \leq N} \sqrt{\beta^{2}+\left|\nabla^{+,+} u(i, j)\right|^{2}}, \quad \beta \geq 0 .
$$

Notice that when $\beta=0, J_{d}^{0}$ coincides with the discrete total variation.

For any function $w \in L^{2}\left(\Omega_{N}\right)$, its Fourier coefficients are

$$
\hat{w}_{\frac{l}{N}, \frac{j}{N}}=\int_{\Omega_{N}} w(x, y) e^{-2 \pi i \frac{(l x+j y)}{N}} \quad \text { for }(l, j) \in \mathbb{Z}^{2} .
$$

As in [24], our plan is to compute a band limited approximation to the solution of the restoration problem for (1). For that we define

$$
\mathcal{B}:=\left\{u \in L^{2}\left(\Omega_{N}\right): \hat{u} \text { is supported in }\left\{-\frac{1}{2}+\frac{1}{N}, \ldots, \frac{1}{2}\right\}^{2}\right\} .
$$

We notice that $\mathcal{B}$ is a finite dimensional vector space of dimension $N^{2}$ which can be identified with $X$. Both $J(u)=\int_{\Omega_{N}}|D u|$ and $J_{d}^{0}(u)$ are norms on the quotient space $\mathcal{B} / \mathbb{R}$, hence they are equivalent. With a slight abuse of notation we indistinctly write $u \in \mathcal{B}$ or $u \in X$.

From now on, $i$ will denote an integer in $\{1, \ldots, N\}$ and not the complex number $\sqrt{-1}$.

We assume that the convolution kernel $h \in L^{2}\left(\Omega_{N}\right)$ is such that $\hat{h}$ is supported in $\left\{-\frac{1}{2}+\frac{1}{N}, \ldots, \frac{1}{2}\right\}^{2}$ and $\hat{h}(0,0)=1$. If $u \in \mathcal{B}$, then we can compute $h * u$ using the Fourier transform

$$
\widehat{h * u}\left(\frac{i}{N}, \frac{j}{N}\right)=\hat{h}\left(\frac{i}{N}, \frac{j}{N}\right) \hat{u}\left(\frac{i}{N}, \frac{j}{N}\right) .
$$

Let $G \in \ell^{\infty}\left(\mathbb{Z}^{2}\right)$ be a discrete convolution kernel periodic of period $N$. Assume that $G(i, j)>0$ for all $(i, j) \in \mathbb{N} \times \mathbb{N}$. As we mentioned in the introduction, we propose to incorporate the image acquisition model (1) as a set of local constraints

$$
G *(h * u-z)(i, j) \leq \bar{\sigma}^{2}, \quad \forall(i, j) \in\{1, \ldots, N\} .
$$

Notice that we have used the value $\bar{\sigma}>0$ as an estimate of the standard deviation of the noise $\sigma$. We will make tests with $\bar{\sigma}=\sigma$ and also with values of $\bar{\sigma}$ different from $\sigma$. The effective support of $G$ must permit the statistical estimation of the variance of the noise with (17). Then we minimize the functional $J_{d}^{\beta}(u)$ on $X$ subject to the family of constraints (11). Thus, we propose to solve the optimization problem:

$$
\begin{aligned}
& \min _{u \in \mathcal{B}} J_{d}^{\beta}(u) \\
& \text { subject to } G *(h * u-z)^{2}(i, j) \leq \bar{\sigma}^{2} \quad \forall(i, j) .
\end{aligned}
$$

Now, we observe that (18) can be solved with a gradient descent approach and Uzawa's method. Indeed, to guarantee that the assumptions of Uzawa's method [16] hold we use a gradient descent strategy. For that, let $v \in X$ and $\gamma>0$. At each step we have to solve a problem like

$$
\begin{aligned}
& \min _{u \in \mathcal{B}} \gamma|u-v|_{X}^{2}+J_{d}^{\beta}(u) \\
& \text { subject to } G *(h * u-z)^{2}(i, j) \leq \bar{\sigma}^{2} \quad \forall(i, j) .
\end{aligned}
$$


Lemma 1 ( $i$ ) For any $\gamma, \beta \geq 0$, problem (19) has a solution. If $\gamma>0$, the solution is unique.

(ii) If $\gamma=0, \beta>0$, and we assume that $\hat{h}(0,0) \neq 0, \inf _{c \in \mathbb{R}} G *(z-c)^{2}>\bar{\sigma}^{2}$, then the solution of (18) is unique. If $\gamma=\beta=0$, the previous assumptions hold and $u$ is a solution of (18), then $h * u$ is unique.

Proof. (i) If $u_{n}$ is a minimizing sequence, then $\nabla^{+,+} u_{n}$ is bounded in $Y$. Since $G *\left(h * u_{n}-z\right)^{2} \leq \bar{\sigma}^{2}$, and $G>0$, then $h * u_{n}\left(i_{0}, j_{0}\right)$ is bounded for some $\left(i_{0}, j_{0}\right)$. Since $h \geq 0$ and is not identically null, then $u_{n}(i, j)$ is bounded for some $(i, j)$. This implies that $u_{n}$ is bounded in $X$. Since the functional and the constraints are convex, (18) has a solution. If $\gamma>0$, the functional is strictly convex and the solution is unique.

(ii) Let $u_{1}, u_{2} \in X$ be two solutions of (18), and let $\bar{u}=\frac{u_{1}+u_{2}}{2}$. We have

$$
(h * \bar{u}(i, j)-z(i, j))^{2} \leq \frac{\left(h * u_{1}(i, j)-z(i, j)\right)^{2}}{2}+\frac{\left(h * u_{2}(i, j)-z(i, j)\right)^{2}}{2} \quad \forall(i, j) .
$$

If the equality in $(20)$ holds for all $(i, j)$, then $h * u_{1}=h * u_{2}$. If the inequality is strict for some $\left(i_{0}, j_{0}\right)$ then

$$
G *(h * \bar{u}(i, j)-z(i, j))^{2}<\frac{G *\left(h * u_{1}(i, j)-z(i, j)\right)^{2}}{2}+\frac{G *\left(h * u_{2}(i, j)-z(i, j)\right)^{2}}{2}<\bar{\sigma}^{2} \quad \forall(i, j) .
$$

Let $t \in[0,1)$ and $u_{t}=t \bar{u}$. Then since $f(t):=G *\left(h * u_{t}(i, j)-z(i, j)\right)^{2}$ is continuous in $t$, then $f(t)<\bar{\sigma}^{2}$ for $t$ near 1. In other words, $u_{t}$ satisfies the constraints in (18). Now, observe that $J_{d}^{\beta}\left(u_{t}\right) \leq J_{d}^{\beta}(\bar{u})$ and the inequality is strict unless $\nabla^{+,+} \bar{u}=0$. Since $\bar{u}$ is a minimum of $(18)$, we have that $\nabla^{+,+} \bar{u}=0$, hence $\bar{u}(i, j)=c$ for some constant $c \in \mathbb{R}$. Now, we have $G *(h * \bar{u}-z)^{2}=G *(c-z)^{2} \leq \bar{\sigma}^{2}$, contradicting our assumptions. We conclude that $h * u_{1}=h * u_{2}$. Now, if $\beta>0, J_{d}^{\beta}$ is strictly convex, and this implies that $\nabla^{+,+} u_{1}=\nabla^{+,+} u_{2}$, and, thus, $u_{1}=u_{2}+\tilde{c}$ for some constant $\tilde{c}$. Recalling that $h * u_{1}=h * u_{2}$, this implies that $u_{1}=u_{2}$.

Remark 2 The result in $(i i)$ also holds if we add the constraint $\hat{u}(0,0)=\hat{z}(0,0)$ and we minimize $(21)$ in $\{u \in \mathcal{B}: \hat{u}(0,0)=\hat{z}(0,0)\}$. In that case, we define $u_{t}=t \bar{u}+c_{t}, t \in[0,1)$, where $c_{t}:=(1-t) \hat{z}(0,0)$ and we observe that $\hat{u}_{t}(0,0)=\hat{z}(0,0)$. Again, since $f(t):=G *\left(h * u_{t}(i, j)-z(i, j)\right)^{2}$ is continuous in $t$, then $f(t)<\bar{\sigma}^{2}$ for $t$ near 1 . In other words, $u_{t}$ satisfies both constraints, the one in (18) and $\hat{u}_{t}(0,0)=\hat{z}(0,0)$.

We solve (19) using the unconstrained formulation

$$
\min _{u \in X} \max _{\lambda \geq 0} \mathcal{L}^{\gamma}(u,\{\lambda\} ; v)
$$

where $\lambda=(\lambda(i, j))_{i, j=1}^{N}$ and

$$
\mathcal{L}^{\gamma}(u,\{\lambda\} ; v)=\gamma|u-v|_{X}^{2}+J_{d}^{\beta}(u)+\sum_{i, j=1}^{N} \lambda(i, j)\left(G *(h * u-z)^{2}(i, j)-\bar{\sigma}^{2}\right) .
$$

Since, by Lemma 1, problem (19) has a solution, the classical existence result of saddle points (see [16]) proves the existence of a solution of (21). Indeed the following result is classical and can be found, for instance, in [16] (Theorems 4 and 5, pp. 59-60). 
Theorem 1 Assume that $z \in h * \mathcal{B}$. Then for any $\beta, \gamma \geq 0$ there is a solution $(u,\{\lambda\})$ of (21), i. e., a saddle point $(u,\{\lambda\})$ of $\mathcal{L}^{\gamma}(\cdot, \cdot ; v)$. If $(u,\{\lambda\})$ is a solution of (21), then $u$ is a solution of (19).

Remark 3 If $z \in \overline{h * \mathcal{B}}$ then $z \in h * \mathcal{B}$. Indeed, if $z \in \overline{h * \mathcal{B}}$, there is a sequence $u_{n} \in \mathcal{B}$ such that $z_{n}:=h * u_{n} \rightarrow z$ in $L^{2}\left(\Omega_{N}\right)$. Since $\operatorname{supp}\left(\hat{z}_{n}\right) \subseteq S:=\left\{(r, s) \in\left\{-\frac{1}{2}+\frac{1}{N}, \ldots, \frac{1}{2}\right\}^{2}: \hat{h}(r, s) \neq 0\right\}$, also $\operatorname{supp}(\hat{z}) \subseteq S$. In that case we may solve the equation $\hat{h} \hat{u}=\hat{z}$ so that $u \in \mathcal{B}$.

We prove that the saddle point can be computed using Uzawa's algorithm. For that we need the following result whose proof is immediate.

Theorem 2 Assume that $\beta, \gamma \geq 0$, and $G(i, j)>0$ for any $(i, j) \in \mathbb{N} \times \mathbb{N}$. Assume that $z \in h * \mathcal{B}$. Then there is a solution $u$ of

$$
\min _{u \in X} \mathcal{L}^{\gamma}(u,\{\lambda\} ; v)
$$

for any values of $\lambda(i, j) \geq 0, i, j \in\{0, \ldots, N-1\}$. If $\gamma>0$, then the solution of (22) is unique.

Remark 4 Even if not needed in the sequel, let us observe that uniqueness also holds if $\gamma=0$ in the following form:

a) If $\gamma=0, \beta>0, \lambda=0$, then any two solutions differ in a constant. Hence, the solution is unique if we minimize $(21)$ in $\{u \in \mathcal{B}: \hat{u}(0,0)=\hat{z}(0,0)\}$.

b) If $\gamma=0, \beta>0, \lambda$ is not identically zero, then the solution is unique.

c) If $\gamma=\beta=0$ and $\lambda \geq 0$ is not identically null, then $h * u$ is unique (thus $u$ is unique if $H u:=h * u$ is injective).

d) If $\gamma=\beta=0$ and $\lambda=0$, then $u$ is a constant which is unique if we impose $\hat{u}(0,0)=\hat{z}(0,0)$.

We assume that $\beta \geq 0, \gamma>0$. We solve (19) with the Uzawa's algorithm.

\section{Algorithm: TV based restoration algorithm with local constraints}

1. Set $u^{0}=0$ or, better, $u^{0}=z$. Set $n=0$.

2. Use Uzawa's algorithm to solve the problem

$$
\min _{u \in X} \max _{\lambda \geq 0} \mathcal{L}^{\gamma}\left(u,\{\lambda\} ; u^{n}\right)
$$

that is:

(a) Choose any set of values $\lambda^{0}(i, j) \geq 0,(i, j) \in\{1, \ldots, N\}^{2}$, and $u_{0}^{n}=u^{n}$.

Iterate from $p=0$ until convergence of $\lambda^{p}$ the following steps:

(b) With the values of $\lambda^{p}$ solve:

$$
\min _{u} \mathcal{L}^{\gamma}\left(u,\left\{\lambda^{p}\right\} ; u^{n}\right)
$$

starting with the initial condition $u_{p}^{n}$. Let $u_{p+1}^{n}$ be the solution obtained.

(c) Update $\lambda$ in the following way:

$$
\lambda^{p+1}(i, j)=\max \left(0, \lambda^{p}(i, j)+\rho\left(G *\left(h * u_{p+1}^{n}-z\right)^{2}(i, j)-\bar{\sigma}^{2}\right)\right) \quad \forall(i, j) .
$$

3. Let $u^{n+1}$ be the solution of (23). Stop iterations on $n$ when convergence of $u^{n}$.

Proposition 1 Assume that $z \in h * \mathcal{B}, \gamma>0$. Then Uzawa's algorithm converges. 
As usual, we denote by $\|v\|_{q}=\left(\sum_{i, j=1}^{N}|v(i, j)|^{q}\right)^{1 / q}$ for any $v \in X, 1 \leq q<\infty$. We denote $\|v\|_{\infty}=\max _{(i, j) \in\{1, \ldots, N\}}|v(i, j)|$.

Proof. Let us write $R(u)=G *(h * u-z)^{2}$. Since $\gamma>0$, the assumptions of Uzawa's algorithm [16] are satisfied once we prove that $R(u)$ is Lipschitz on bounded sets of $X$ and we observe that the sequence $u_{p}$ constructed in Step 2 (and denoted $u_{p}^{n}$ there) of the above algorithm is bounded in $X$. Assume that $U \subseteq X$ is a bounded set. Let $u, \bar{u} \in U$. Since $\|G\|_{1} \leq 1$, we have

$$
\begin{aligned}
\|R(u)-R(\bar{u})\| & \leq\|G\|_{1}\left\|(h * u-z)^{2}-(h * \bar{u}-z)^{2}\right\|_{X} \\
& \leq 2\|z\|_{\infty}\|h *(u-\bar{u})\|_{X}+\|h *(u+\bar{u})\|_{\infty}\|h *(u-\bar{u})\|_{X} \\
& \leq D\|u-\bar{u}\|_{X}
\end{aligned}
$$

where $D$ is a constant depending on the norms of $h$ and $z$ and on the bound for $U$. Now, to prove that $\left\{u_{p}\right\}_{p}$ is bounded we observe that

$$
\mathcal{L}^{\gamma}\left(u_{p},\left\{\lambda^{p}\right\} ; v\right) \leq \mathcal{L}^{\gamma}\left(u,\left\{\lambda^{p}\right\} ; v\right), \quad \forall u \in X,
$$

for all $p$. Choosing $u \in X$ such that $z=h * u$, we obtain that

$$
\gamma\left\|u_{p}-v\right\|_{X}^{2}+J_{d}^{\beta}\left(u_{p}\right) \leq \gamma\|u-v\|_{X}^{2}+J_{d}^{\beta}(u)
$$

hence $\left\{u_{p}\right\}_{p}$ is bounded in $X$. Now, the Proposition follows from Theorem 5 in [16], Sect. 3.1.

To justify the Algorithm, we prove the convergence of $u^{n}$ as $n \rightarrow \infty$ to a solution of (18). This type of result was proved in the continuous case by L. Vese in [34], Theorem 5.4. Our proof will be based on the techniques in [9].

Proposition 2 Let $\beta \geq 0, \gamma>0$. Assume that $u^{0} \in X$ satisfies the constraints. Then $u^{n}$ tends to a solution $u$ of (18) as $n \rightarrow \infty$.

Proof. Let us write $\gamma=\frac{1}{2 \Delta t}, \Delta t>0$. Let $C$ be the convex set of functions $u \in \mathcal{B}$ such that $G *(h * u-z)^{2}(l, j) \leq \bar{\sigma}^{2} \forall(l, j)$. Let $\delta_{C}(u)=0$ if $u \in C$ and $+\infty$ otherwise. Then for each $n u^{n}$ is a solution of

$$
\frac{u^{n+1}-u^{n}}{\Delta t}+\partial J_{d}^{\beta}\left(u^{n+1}\right)+\partial \delta_{C}\left(u^{n+1}\right) \ni 0
$$

where we write the inclusion symbol $\ni$ instead of the equality since $\partial \delta_{C}(u)$ and $\partial J_{d}^{0}(u)$ may be multivalued. Let $\bar{u} \in \mathcal{B}$ be a solution of the stationary problem (18). Then we have

$$
\partial J_{d}^{\beta}(\bar{u})+\partial \delta_{C}(\bar{u}) \ni 0
$$

and, therefore,

$$
u^{n+1}-\bar{u}+\Delta t\left(\partial J_{d}^{\beta}\left(u^{n+1}\right)-\partial J_{d}^{\beta}(\bar{u})\right)+\Delta t\left(\partial \delta_{C}\left(u^{n+1}\right)-\partial \delta_{C}(\bar{u}) \ni u^{n}-\bar{u} .\right.
$$

Multiplying by $u^{n+1}-\bar{u}$ and using that both $\partial J_{d}$ and $\partial \delta_{C}$ are maximal monotone operators, we have

$$
\left\|u^{n+1}-\bar{u}\right\|_{X}^{2} \leq\left\langle u^{n}-\bar{u}, u^{n+1}-\bar{u}\right\rangle_{X} \leq\left\|u^{n}-\bar{u}\right\|_{X}\left\|u^{n+1}-\bar{u}\right\|_{X}
$$

hence

$$
\left\|u^{n+1}-\bar{u}\right\|_{X} \leq\left\|u^{n}-\bar{u}\right\|_{X}
$$


and, thus, $u^{n}$ is bounded in $X$. Since $u^{n}$ is bounded in $X$, we may extract a subsequence $u^{n_{j}}$ such that $u^{n_{j}}$ converges to some function $u^{*}$ in $X$.

Now, multiplying (24) by $\frac{u^{n+1}-u^{n}}{\Delta t}$, taking scalar products, and adding the identities obtained we have

$$
\begin{aligned}
\sum_{n=0}^{p}\left\|\frac{u^{n+1}-u^{n}}{\Delta t}\right\|_{X}^{2} & =\frac{1}{\Delta t} \sum_{n=0}^{p}\left\langle\partial\left(J_{d}^{\beta}+\delta_{C}\right)\left(u^{n+1}\right), u^{n}-u^{n+1}\right\rangle_{X} \\
& \leq \frac{1}{\Delta t} \sum_{n=0}^{p}\left(\left(J_{d}^{\beta}+\delta_{C}\right)\left(u^{n}\right)-\left(J_{d}^{\beta}+\delta_{C}\right)\left(u^{n+1}\right)\right) .
\end{aligned}
$$

Hence,

$$
\Delta t \sum_{n=0}^{p}\left\|\frac{u^{n+1}-u^{n}}{\Delta t}\right\|_{X}^{2}+\left(J_{d}^{\beta}+\delta_{C}\right)\left(u^{p+1}\right) \leq\left(J_{d}^{\beta}+\delta_{C}\right)\left(u^{0}\right) .
$$

This estimate implies that $u^{n+1}-u^{n} \rightarrow 0$ as $n \rightarrow \infty$.

Letting $n \rightarrow \infty$ in (24) along the sequence $n_{j}$ we have that $u^{*}$ is a solution of

$$
\partial J_{d}^{\beta}(u)+\partial \delta_{C}(u) \ni 0 .
$$

In other words, $u^{*}$ is a solution of DP. Replacing $\bar{u}$ by $u^{*}$ in (26), we have

$$
\left\|u^{n+1}-u^{*}\right\|_{X} \leq\left\|u^{n}-u^{*}\right\|_{X}
$$

for all $n$. This implies that $u^{n} \rightarrow u^{*}$ as $n \rightarrow \infty$.

It remains to explain the algorithm used to solve problem (23) in Step 2.(b) of the above Algorithm. For that, observe that the Euler-Lagrange equation corresponding to (21) is

$$
\frac{u-v}{\Delta t}+\partial J_{d}^{\beta}(u)+h *((G * \lambda)(h * u-z)) \ni 0 .
$$

In our experiments displayed below, we have considered the case $\beta=0$ and we have solved (29) using our extension of Chambolle's algorithm [10] to the restoration case given in [3].

We have also experimented with the case $\beta>0$ with comparable results (we shall display one experiment with $\beta=1$ in Figure 5). To give the numerical scheme for the case $\beta>0$, let us define the discrete divergence operator. For that, we use the Euclidean scalar product in $Y$ is defined in the standard way by

$$
\langle\vec{p}, \vec{q}\rangle_{Y}=\sum_{1 \leq i, j \leq N}\left(p_{i, j}^{1} q_{i, j}^{1}+p_{i, j}^{2} q_{i, j}^{2}\right)
$$

for every $\vec{p}=\left(p^{1}, p^{2}\right), \vec{q}=\left(q^{1}, q^{2}\right) \in Y$. The norm of $\vec{p}=\left(p^{1}, p^{2}\right) \in Y$ is, as usual, $\|\vec{p}\|_{Y}=\langle\vec{p}, \vec{p}\rangle_{Y}^{1 / 2}$. By analogy with the continuous setting, we introduce a discrete divergence $\operatorname{div}^{-,-}$as the dual operator of $\nabla^{+,+}$, i.e., for every $\vec{p} \in Y$ and $u \in X$ we have

$$
\left\langle-\operatorname{div}^{-,-} \vec{p}, u\right\rangle_{X}=\left\langle\vec{p}, \nabla^{+,+} u\right\rangle_{Y}
$$


One can easily check that $\operatorname{div}^{-,-}$is given by $[10]$

$$
\begin{aligned}
\operatorname{div}^{-,} \vec{p}(i, j) & =\left\{\begin{array}{ccc}
p^{1}(i, j)-p^{1}(i-1, j) & \text { if } & 1<i<N \\
p^{1}(i, j) & \text { if } & i=1 \\
-p^{1}(i-1, j) & \text { if } & i=N
\end{array}\right. \\
& +\left\{\begin{array}{ccc}
p^{2}(i, j)-p^{2}(i, j-1) & \text { if } & 1<j<N \\
p^{2}(i, j) & \text { if } & j=1 \\
-p^{2}(i, j-1) & \text { if } & j=N
\end{array}\right.
\end{aligned}
$$

for every $\vec{p}=\left(p^{1}, p^{2}\right) \in Y$.

For $\beta>0$ the $\ni \operatorname{sign}$ in $(29)$ becomes an equality, $\partial J_{d}^{\beta}(u)=\operatorname{div}^{-,-}\left(\frac{\nabla^{+,+} u}{\sqrt{\beta^{2}+\left|\nabla^{+,+} u\right|^{2}}}\right)$ for any $u \in X$ and we consider the following iterative scheme

$$
A u^{m+1}=v+\Delta t h *((G * \lambda) z)+\Delta t \operatorname{div}^{-,-}\left(\frac{\nabla^{+,+} u^{m}}{\sqrt{\beta^{2}+\left|\nabla^{+,+} u^{m}\right|^{2}}}\right) \quad \text { in } X
$$

where

$$
A u=u+\Delta t h *((G * \lambda) h * u),
$$

and any $u^{0} \in X, m \geq 0$. Observe that $A$ is a positive symmetric linear operator on $X$ whose inverse $A^{-1}$ is a contraction in $X$, hence satisfies $\left\|A^{-1}\right\| \leq 1$ for any $\lambda \geq 0$ where $\left\|A^{-1}\right\|$ denotes the norm of $A^{-1}$ in $X$. In particular, (31) can be solved by means of a conjugate gradient algorithm. Now observe that there is a unique solution $u^{*}$ of $(29)$. Then

$$
\begin{aligned}
\left\|u^{m+1}-u^{*}\right\|_{X} & \leq \Delta t\left\|\operatorname{div}^{-,-}\left(\frac{\nabla^{+,+} u^{m}}{\sqrt{\beta^{2}+\left|\nabla^{+,+} u^{m}\right|^{2}}}\right)-\operatorname{div}^{-,-}\left(\frac{\nabla^{+,+} u^{*}}{\sqrt{\beta^{2}+\left|\nabla^{+,+} u^{*}\right|^{2}}}\right)\right\|_{X} \\
& \leq \sqrt{8} \Delta t\left\|\frac{\nabla^{+,+} u^{m}}{\sqrt{\beta^{2}+\left|\nabla^{+,+} u^{*}\right|^{2}}}-\frac{\nabla^{+,+} u^{*}}{\sqrt{\beta^{2}+\left|\nabla^{+,+} u^{*}\right|^{2}}}\right\|_{Y} .
\end{aligned}
$$

since

$$
\|\operatorname{div} \xi\|_{X}^{2} \leq 8\|\xi\|_{Y}^{2} \quad \forall \xi \in Y
$$

Finally, since

$$
\left\|\frac{\xi}{\sqrt{\beta^{2}+|\xi|}}-\frac{\xi^{\prime}}{\sqrt{\beta^{2}+\left|\xi^{\prime}\right|}}\right\|_{Y} \leq \frac{1}{\beta}\left\|\xi-\xi^{\prime}\right\|_{Y} \quad \forall \xi, \xi^{\prime} \in Y
$$

and

$$
\left\|\nabla^{+,+} u\right\|_{Y}^{2} \leq 8\|u\|_{X}^{2} \quad \forall u \in X
$$

we have

$$
\left\|u^{m+1}-u^{*}\right\|_{X} \leq \frac{8 \Delta t}{\beta}\left\|u^{m}-u^{*}\right\|_{X} \quad \forall m \geq 0 .
$$

Assuming that

$$
\Delta t<\frac{\beta}{8}
$$

we deduce that $u^{m} \rightarrow u^{*}$ as $m \rightarrow \infty$. 
Remark 5 For the sake of completeness, let us point out the following algorithm for the case $\beta>0$, which is due to Bermúdez-Moreno [7]. The Bermúdez-Moreno algorithm for (29) (with $\omega=0$ [7]) can be written as:

$$
\begin{aligned}
& A u^{m}=v+\Delta t h *((G * \lambda) z)+\Delta t \operatorname{div} \xi^{m} \quad \text { in } X \\
& \xi^{m+1}(i, j)=G_{\alpha}\left(\nabla^{+,+} u^{m}(i, j)+\alpha \xi^{m}(i, j)\right), \quad \text { in } \mathbb{R}^{2}, \forall(i, j),
\end{aligned}
$$

where $A$ is given in (32),

$$
\begin{gathered}
G_{\alpha}:=\frac{I-\left(I+\alpha \partial \varphi^{\beta}\right)^{-1}}{\alpha}, \quad \alpha>0, \\
\varphi^{\beta}(\xi)=\sqrt{\beta^{2}+|\xi|^{2}}, \quad \xi \in \mathbb{R}^{2} .
\end{gathered}
$$

In that case, assuming that $\left\{\xi^{m}(i, j)\right\}_{i, j}$ is already known, the first equation of (33) can be solved for $u^{m}$ by means of a conjugate gradient algorithm. Let us write $\xi^{m} \in \mathbb{R}^{2}$ as one of the vectors $\xi^{m}(i, j)$, $i, j \in\{1, \ldots, N\}$ and, for simplicity, write $\nabla^{+,+} u^{m}$ instead of $\nabla^{+,+} u^{m}(i, j)$. Writing

$$
v^{m+1}=\nabla^{+,+} u^{m}+\alpha \xi^{m}-\alpha \xi^{m+1},
$$

and using that $\partial \varphi^{\beta}(v)=\frac{v}{\sqrt{\beta^{2}+|v|^{2}}}$, after some simple computations, the second equation of (33) can be written as

$$
v^{m+1}+\alpha \frac{v^{m+1}}{\sqrt{\beta^{2}+\left|v^{m+1}\right|^{2}}}=\nabla^{+,+} u^{m}+\alpha \xi^{m} .
$$

By squaring both members of this equality, we get

$$
\left|v^{m+1}\right|^{2}+\alpha^{2} \frac{\left|v^{m+1}\right|^{2}}{\beta^{2}+\left|v^{m+1}\right|^{2}}+2 \alpha \frac{\left|v^{m+1}\right|^{2}}{\sqrt{\beta^{2}+\left|v^{m+1}\right|^{2}}}=\left|\nabla^{+,+} u^{m}+\alpha \xi^{m}\right|^{2},
$$

which we solve using a Newton method to find $\left|v^{m+1}\right|^{2}$. Inserting this value in (35), we obtain $v^{m+1}$ and consequently $\xi^{m+1}$ from (34).

Since $\langle A u, u\rangle_{X} \geq\|u\|_{X}^{2}$, if

$$
0<\frac{1}{\alpha}<\frac{2}{\left\|\nabla^{+,+}\right\|^{2}}
$$

where $\left\|\nabla^{+,+}\right\|$denotes the norm of the operator $\nabla^{+,+}: X \rightarrow Y$, the convergence of Bermúdez-Moreno's algorithm was proved in [7], Proposition 3.1 and Corollary 3.2.

\section{Experiments}

First, we simulate a degraded image following the image acquisition model (1). For that we use the modulation transfer function corresponding to SPOT 5 HRG satellite with Hipermode sampling (we refer the reader to [2] for more details):

$$
\hat{h}(\xi, \eta)=e^{-4 \pi \beta_{1}|\xi|} e^{-4 \pi \alpha \sqrt{\xi^{2}+\eta^{2}}} \operatorname{sinc}(2 \xi) \operatorname{sinc}(2 \eta) \operatorname{sinc}(\xi), \quad \xi, \eta \in[-1 / 2,1 / 2],
$$

where $\operatorname{sinc}(\xi)=\sin (\pi \xi) /(\pi \xi), \alpha=0.58$, and $\beta_{1}=0.14$. Then we add to the filtered image a white noise of standard deviation $\sigma=1$. See the reference image and the degraded one in Figure 1.

Since the results obtained are comparable, all the experiments displayed, except Figure 5 where we use $\beta=1$ and the scheme (31), will be done with the restoration model (18) and $\beta=0$. We have solved (29) using our extension of Chambolle's algorithm [10] to the restoration case given in [3]. 

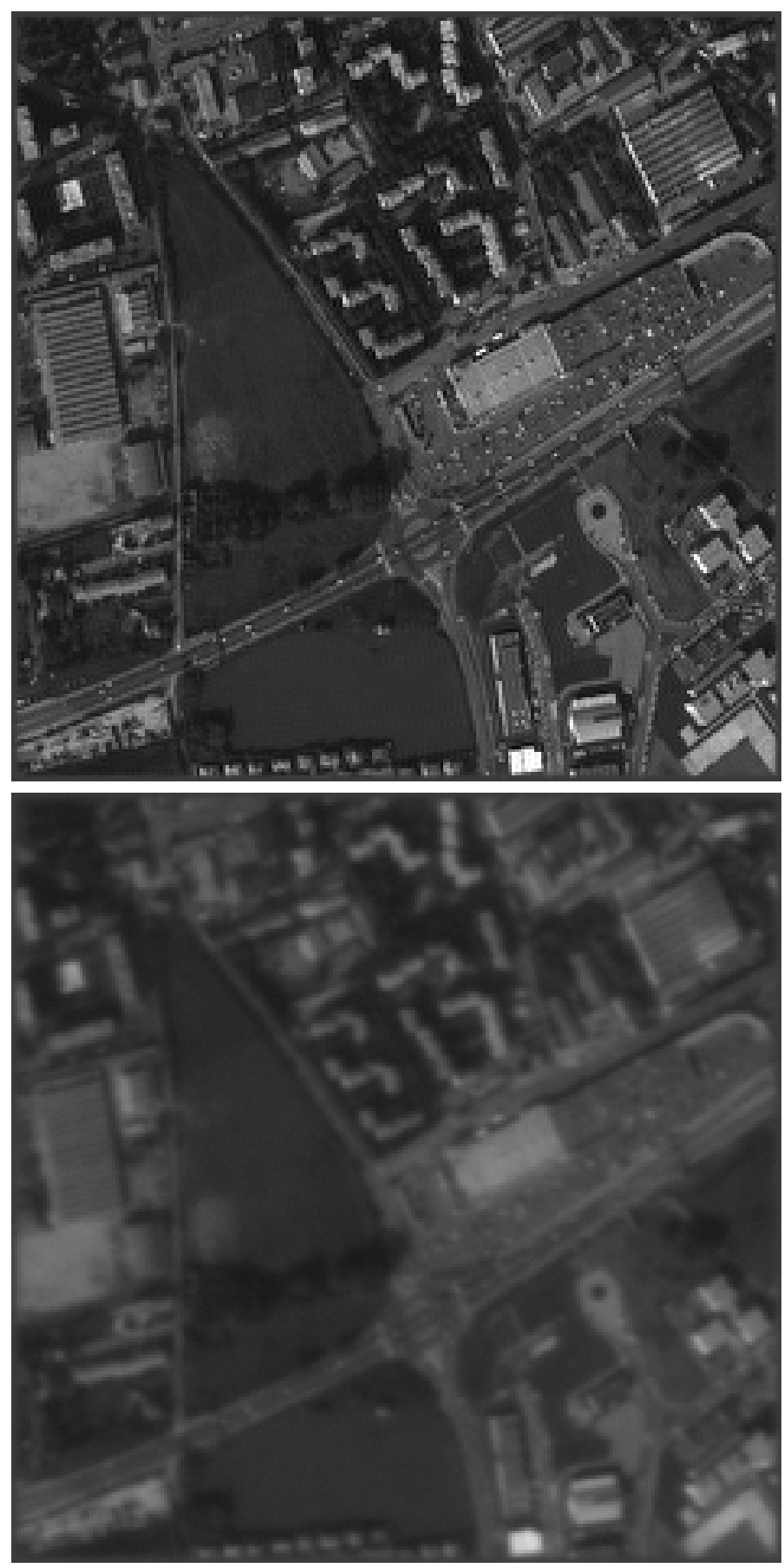

Figure 1: Reference image and a filtered and noised image. The bottom image has been generated applying filter (36) to the top image and adding a white Gaussian noise of standard deviation $\sigma=1$. 
Figure 2 displays two examples of restored images following the discrete version of the restoration model (6), i.e. minimizing the discrete total variation with a global parameter that controls the importance of the data fidelity term. The image in Figure 2.a has been obtained with a parameter $\lambda=10$ that permits to satisfy the constraint $\sum_{i, j=1}^{N}(h * u(i, j)-z(i, j))^{2}=\sigma^{2} N^{2}$. In this case the attained mean value of $(h * u(i, j)-z(i, j))^{2}$ is mean $\left.\left.(h * u-z)^{2}\right)=\operatorname{mean}\left(G *(h * u-z)^{2}\right)\right)=0.9947$. For the purposes of comparison with the case of using local constraints we have variance $\left.\left(G *(h * u-z)^{2}\right)\right)=0.0699$ using a gaussian $G$ with $\sigma_{G}=6.5$. The root mean square error (RMSE) of the image in Figure 2.a is $R M S E=9.4148$. In particular, notice how the structure of the two big textured building is lost. The image in Figure 2.b has been obtained with a parameter $\lambda=40$ which implies a stronger attachment to the data. We recover part of the image textures but noise has been also deconvolved and considered part of the data. In this case, mean $\left.\left.(h * u-z)^{2}\right)=\operatorname{mean}\left(G *(h * u-z)^{2}\right)\right)=0.6327$, variance $\left.\left(G *(h * u-z)^{2}\right)\right)=0.0069$ with a Gaussian $G$ with $\sigma_{G}=6.5$, and $R M S E=9.0970$. Both examples motivate our decision of using local Lagrange multipliers instead of a global one. Indeed, to be able to recover textured regions while denoising smoother ones we need to adapt the Lagrange multiplier to each region.

Figure 3 displays the restoration result obtained using the variational formulation (18) (with as many constraints as pixels) with $\bar{\sigma}^{2}=\sigma^{2}=1$ applied to the image of Figure 1.b. Figure 3.a displays the restored image obtained with a normalized Gaussian window of standard deviation $\sigma_{G}=6.5$. In this case RMSE $=9.0739$, mean $\left(G *(h * u-z)^{2}\right)$ (that is, the average value of $\left.G *(h * u-z)^{2}\right)$ is 0.9669 , and variance $\left.\left(G *(h * u-z)^{2}\right)\right)=0.0012$. Even if we have not imposed the constraint that the noise has zero mean, in other words that the average value of $u$ coincides with the average value of $z$, we have obtained mean $(G *(h * u-z))=0.0014$. Figure 3.b displays the function $\lambda(i, j)$ obtained. We see that the values of $\lambda(i, j)$ are correlated to the geometry of the image.

Figure 4.a displays the residual values $|h * u-z|$ corresponding to the result displayed Figure 2.a. For better visibility, we have re-scaled the residual to have values between 0 and 255 . We see that some structure is present in the residual. Figure 4.b displays the residual values $|h * u-z|$ corresponding to the result displayed in Figure 3.a. We see that in this case the residual has the appearance of noise.

For completeness, Figure 5 displays the restoration result obtained using (18) with $\beta=1, \bar{\sigma}^{2}=\sigma^{2}=$ 1 and $\sigma_{G}=6.5$ applied to the image of Figure 1.b. The numerical scheme used in this case is the scheme (31). In this case RMSE $\left.=9.2323, \operatorname{mean}\left(G *(h * u-z)^{2}\right)=0.9556, \operatorname{variance}\left(G *(h * u-z)^{2}\right)\right)=0.0025$ and mean $(G *(h * u-z))=0.0012$. The result is comparable to the one in Figure 3.a.

Figure 6 displays two more restoration results obtained using our functional (18) with $\bar{\sigma}^{2}=\sigma^{2}=1$ obtained using a Gaussian window of $\sigma_{G}=3$ (Figure 6.a) and $\sigma_{G}=10$ (Figure 6.b). For Figure 6.a we have the values RMSE $=9.0178, \operatorname{mean}\left(G *(h * u-z)^{2}\right)=0.9484$, variance $\left.\left(G *(h * u-z)^{2}\right)\right)=$ $0.0031, \operatorname{mean}(G *(h * u-z))=0.0032$. The corresponding values for Figure 6.b are RMSE $=9.1373$, mean $\left(G *(h * u-z)^{2}\right)=0.9730$, variance $\left.\left(G *(h * u-z)^{2}\right)\right)=0.0006$, mean $(G *(h * u-z))=0.00002$. Even if the results are quite comparable, when the value of $\sigma_{G}$ is small some small noise spots are still present as can be seen in Figure 7.b, which represents a detail of Figure 6.a $\left(\sigma_{G}=3\right)$. Figure 7.a displays the same detail corresponding to Figure 3.a.

Figures 8,9 and 10 display some results obtained with a value of $\bar{\sigma}^{2}$ in the constraint different from the variance of the noise $\sigma^{2}=1$.

In Figure 8 we display the results obtained with $\bar{\sigma}=1.1$. Figure 8.a displays the result obtained when $\sigma_{G}=3$. For this Figure we have the values are RMSE $=9.2279, \operatorname{mean}\left(G *(h * u-z)^{2}\right)=1.1237$, variance $\left.\left(G *(h * u-z)^{2}\right)\right)=0.0073$ and mean $(G *(h * u-z))=0.0034$. Figure 8.b displays the results obtained when $\sigma_{G}=6.5$. In this case, we have RMSE $=9.3193$, mean $\left(G *(h * u-z)^{2}\right)=1.1378$, $\left.\operatorname{variance}\left(G *(h * u-z)^{2}\right)\right)=0.0050$ and mean $(G *(h * u-z))=0.00014$. 

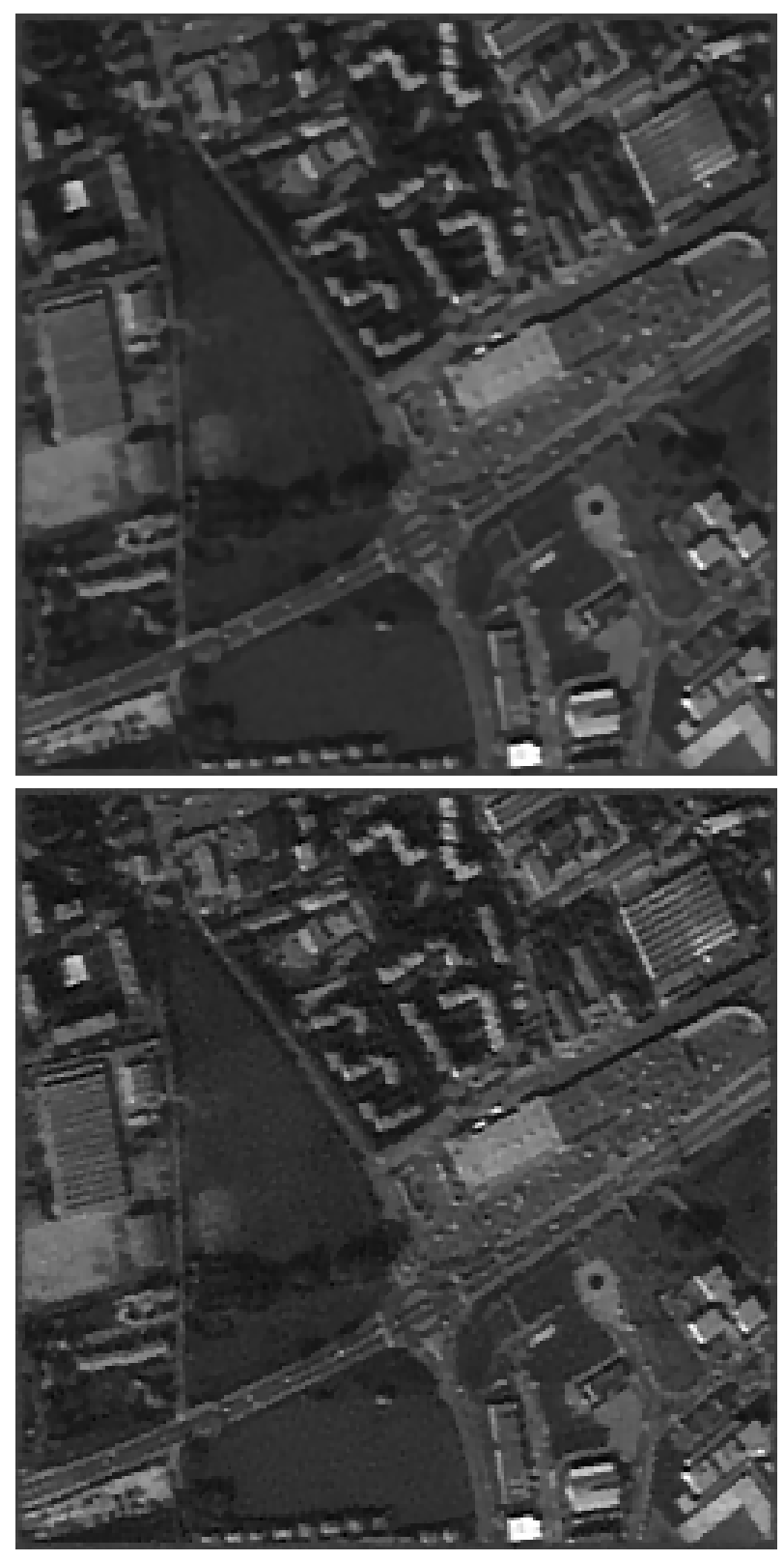

Figure 2: Restored images with a global Lagrange multiplier (with $\beta=0$ ). a) The top image has been obtained using the global parameter $\lambda=10$ which gives a solution that satisfies the constraint $\sum_{i, j=1}^{N}(h * u(i, j)-z(i, j))^{2}=\sigma^{2} N^{2}$. The attained value for this quantity is mean $\left.(h * u-z)^{2}\right)=$ mean $\left.\left(G *(h * u-z)^{2}\right)\right)=0.9947$ and variance $\left.\left(G *(h * u-z)^{2}\right)\right)=0.0699$ using a gaussian $G$ with $\sigma_{G}=6.5$. The RMSE between this result and the reference image is $R M S E=9.4148$. b) The bottom one has been obtained with a global parameter $\lambda=40$ which is bigger than the previous value that permits to satisfy the constraint. In this case, $\left.\left.\operatorname{mean}(h * u-z)^{2}\right)=\operatorname{mean}\left(G *(h * u-z)^{2}\right)\right)=0.6327$, variance $\left.\left(G *(h * u-z)^{2}\right)\right)=0.0069$ with a Gaussian $G$ of $\sigma_{G}=6.5$, and $R M S E=9.0970$. 

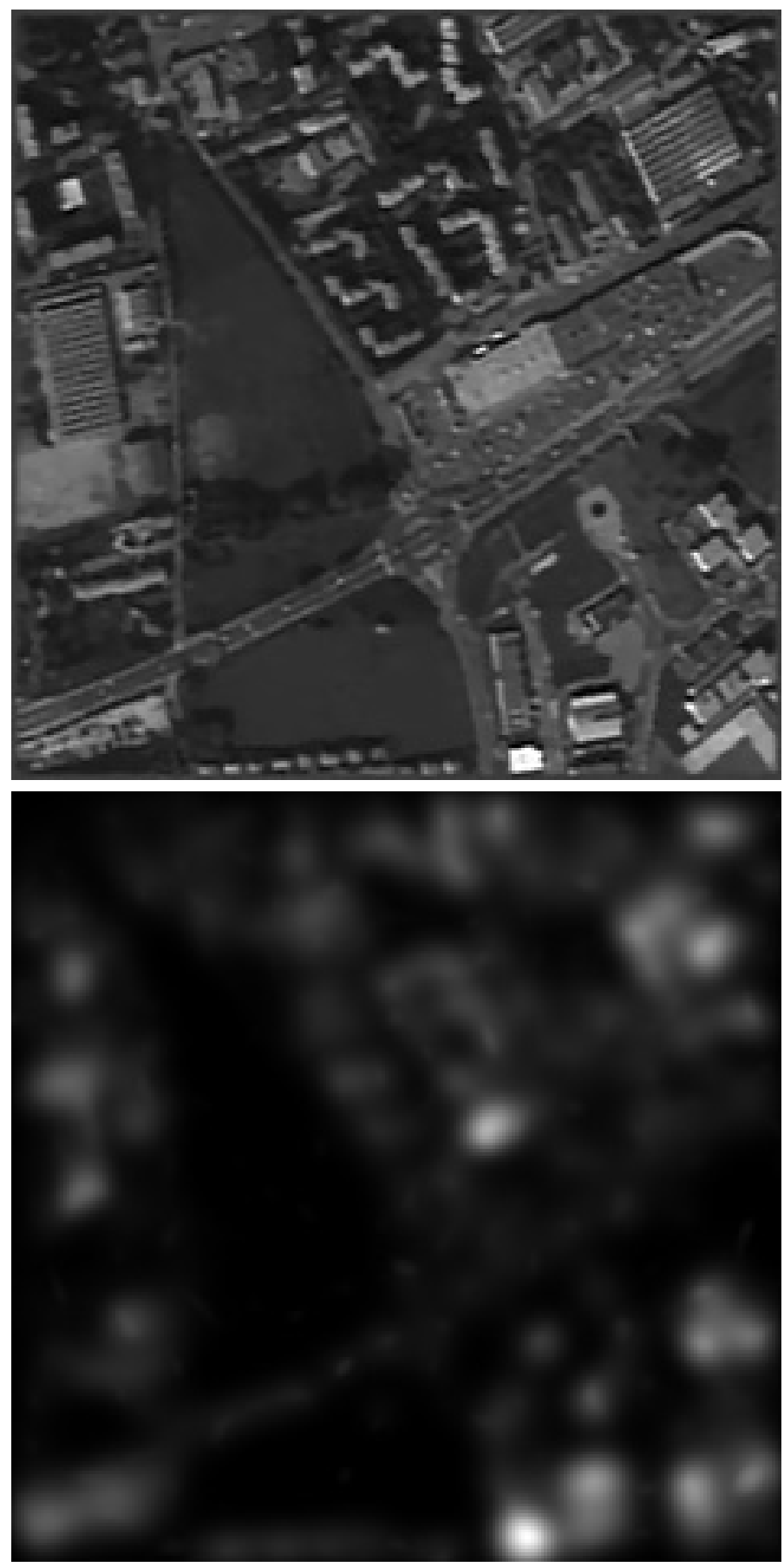

Figure 3: Restored image with local Lagrange multipliers. a) The top image corresponds to the restored image obtained using functional (18) with $\beta=0, \bar{\sigma}^{2}=\sigma^{2}=1$ using a Gaussian window with $\sigma_{G}=6.5$. $\operatorname{RMSE}=9.0739$, mean $\left(G *(h * u-z)^{2}\right)=0.9669$, and $\left.\operatorname{variance}\left(G *(h * u-z)^{2}\right)\right)=0.0012$. b) The bottom one is the function $\lambda(i, j)$ obtained. 

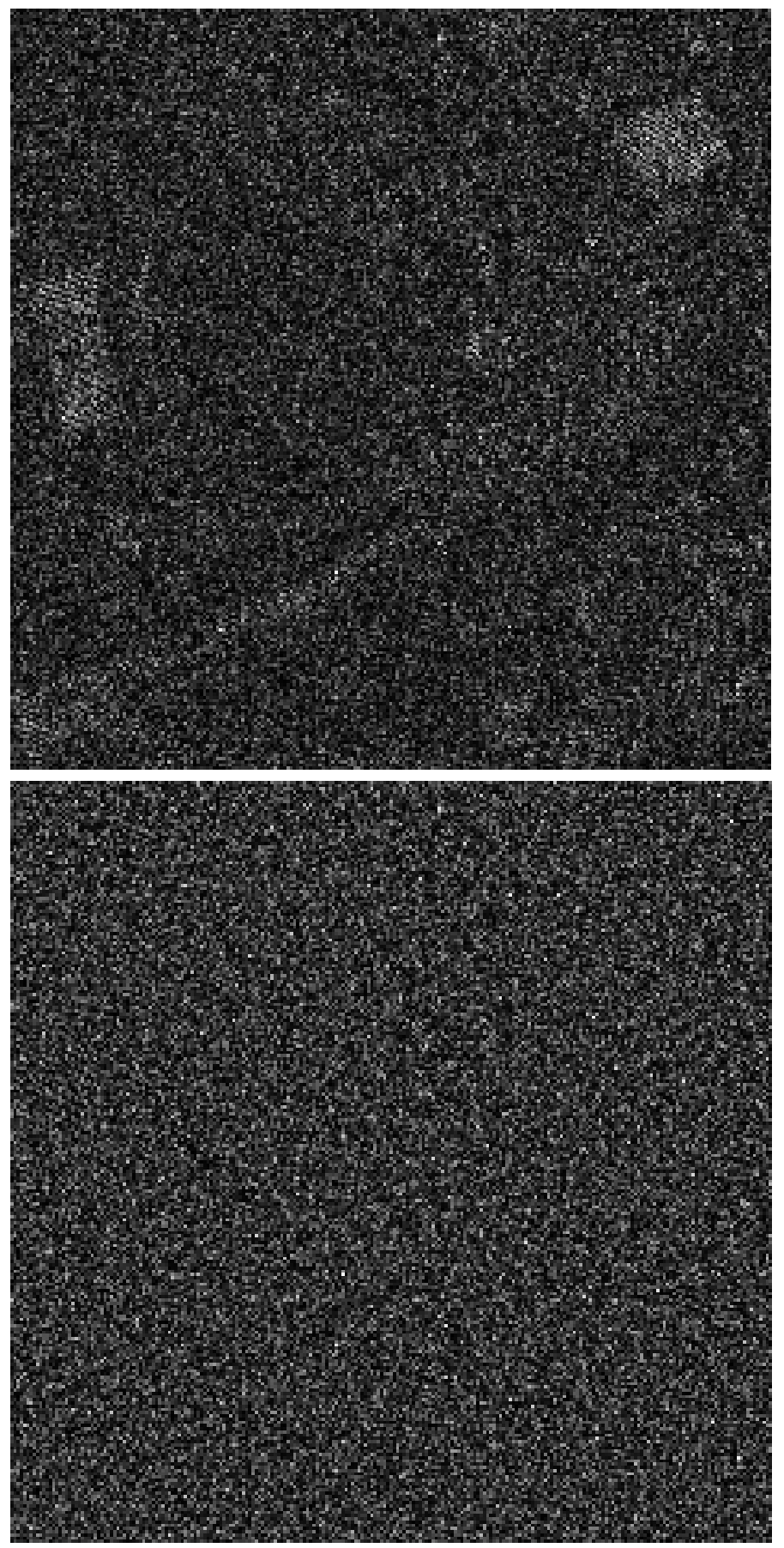

Figure 4: Residual values $h * u-z$. a) The top image displays the residual values $|h * u-z|$, rescaled between 0 and 255, corresponding to the image of Figure 2.a. b) The bottom one displays the residual values corresponding to the image of Figure 3.a. 


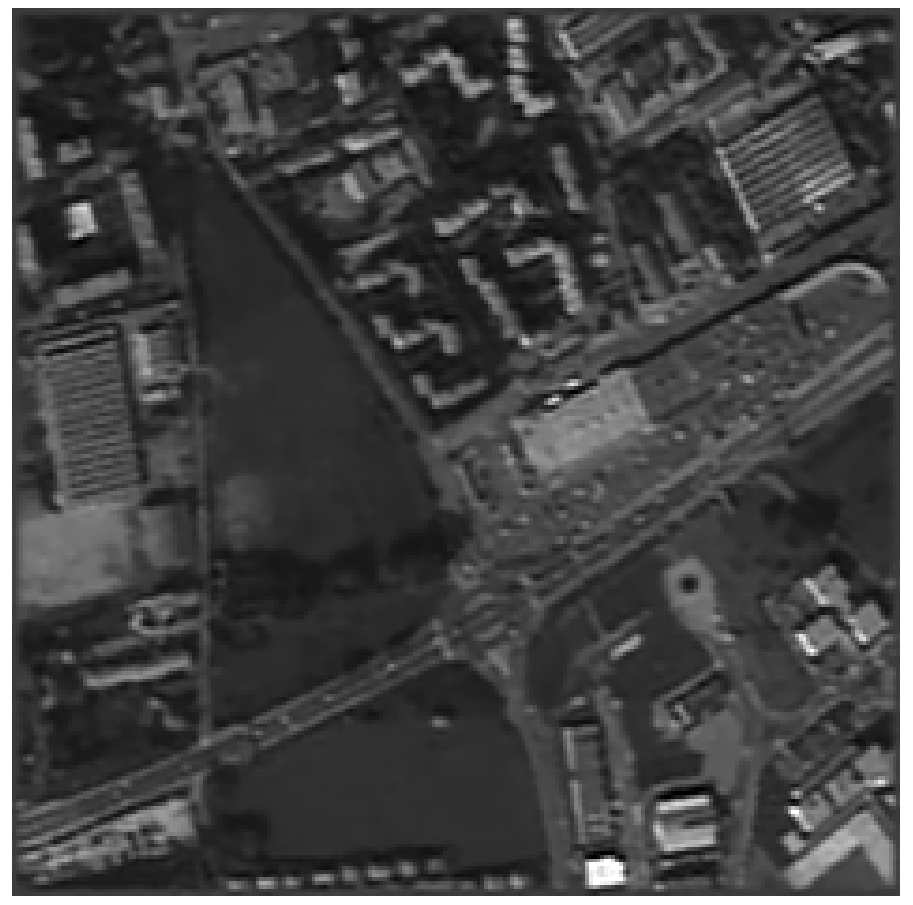

Figure 5: Restored image with local Lagrange multipliers using scheme (31). We display the restored image obtained using functional (18) with $\beta=1, \bar{\sigma}^{2}=\sigma^{2}=1$ and a Gaussian window with $\sigma_{G}=6.5$. The numerical scheme is the one described in (31). We have obtained RMSE $=9.2323, \operatorname{mean}(G *(h *$ $\left.u-z)^{2}\right)=0.9556$, and variance $\left.\left(G *(h * u-z)^{2}\right)\right)=0.0025$. 

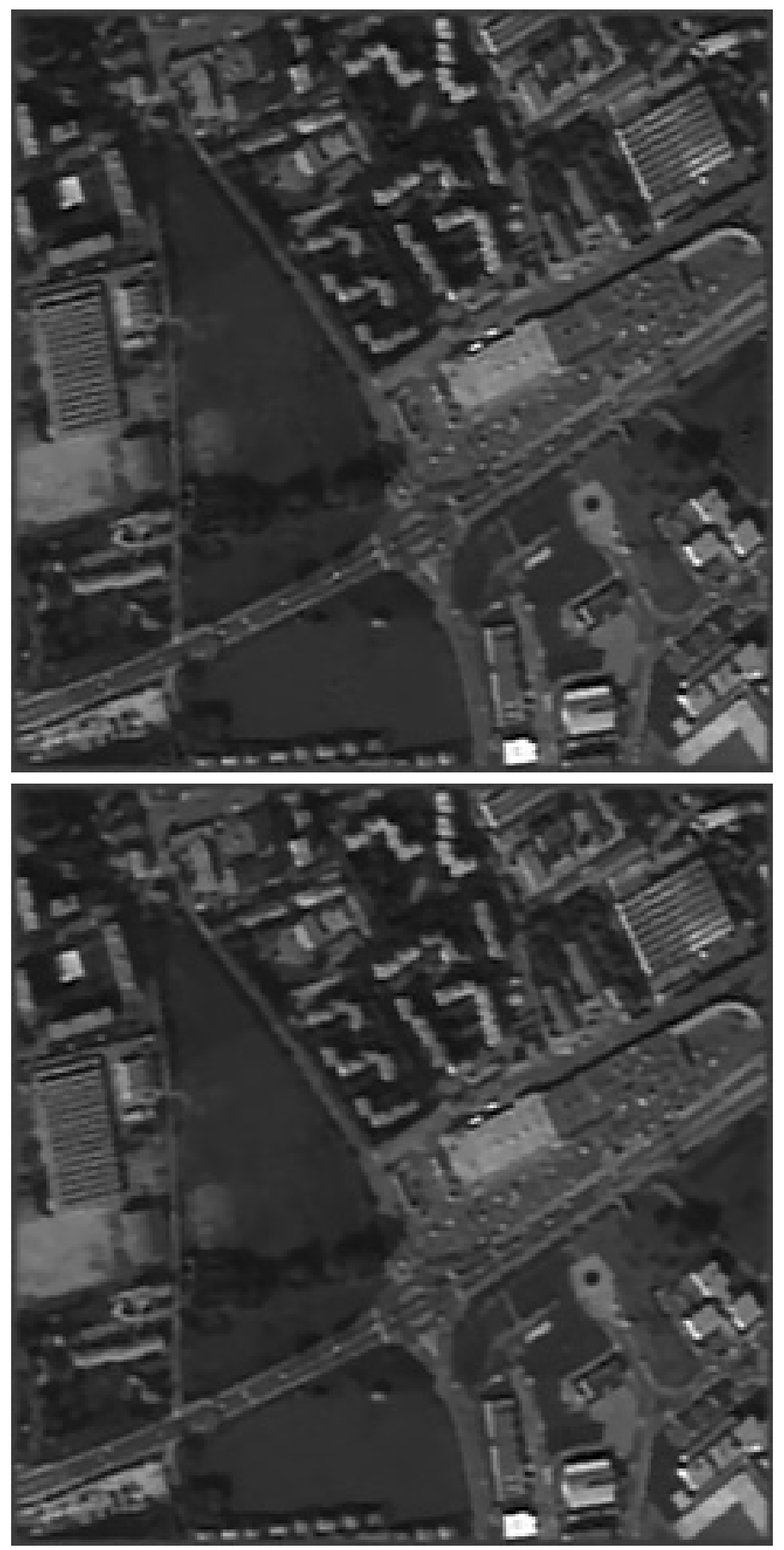

Figure 6: Restored image with local Lagrange multipliers. a) The top image corresponds to the restored image obtained using functional (18) with $\beta=0, \bar{\sigma}^{2}=\sigma^{2}=1$ using a Gaussian window with $\sigma_{G}=3$. The RMSE $=9.0178, \operatorname{mean}\left(G *(h * u-z)^{2}\right)=0.9484$, and variance $\left.\left(G *(h * u-z)^{2}\right)\right)=0.0031$. b) The bottom image is the restored image obtained using $\beta=0, \bar{\sigma}^{2}=\sigma^{2}=1, \sigma_{G}=10$. In this case, we have $\operatorname{RMSE}=9.1373, \operatorname{mean}\left(G *(h * u-z)^{2}\right)=0.9730$, and $\left.\operatorname{variance}\left(G *(h * u-z)^{2}\right)\right)=0.0006$. 

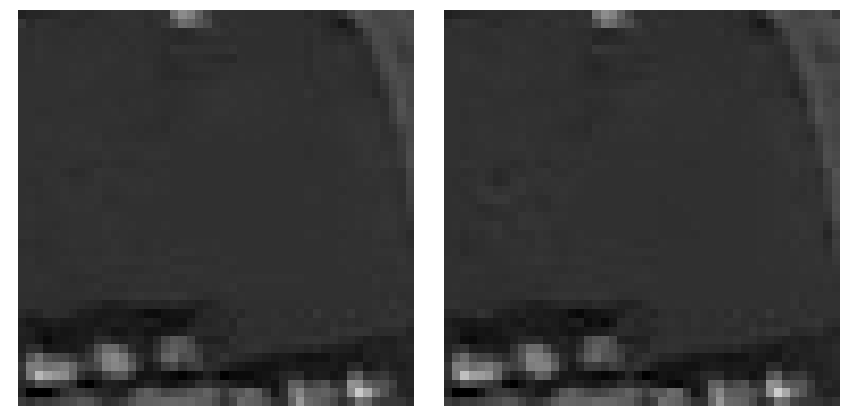

Figure 7: A detail of Figures 3.a and 6.a. a) We display a detail corresponding to an enhanced version of Figures 3.a. b) We display a detail corresponding to an enhanced version of Figures 6.a. The results are comparable but we see a slight perturbation in the left centered part of the right image.

In Figure 9 we have taken $\bar{\sigma}=1.2$. In Figure 9. a we display the result obtained when $\sigma_{G}=3$. For this Figure we have the values are RMSE $=9.4652, \operatorname{mean}\left(G *(h * u-z)^{2}\right)=1.3019$, variance $\left.\left(G *(h * u-z)^{2}\right)\right)=$ 0.0196 and $\operatorname{mean}(G *(h * u-z))=0.00015$. Figure 9.b displays the results obtained when $\sigma_{G}=6.5$. In this case, we have RMSE $=9.5763, \operatorname{mean}\left(G *(h * u-z)^{2}\right)=1.3228$, variance $\left.\left(G *(h * u-z)^{2}\right)\right)=0.0163$ and $\operatorname{mean}(G *(h * u-z))=-0.0020$.

Figure 10 displays some results obtained taking the value $\bar{\sigma}=0.9$. Figures 10.a display the results obtained when $\sigma_{G}=6.5$. In this case, we have the values $\operatorname{RMSE}=8.9157, \operatorname{mean}\left(G *(h * u-z)^{2}\right)=$ 0.7935 , variance $\left.\left(G *(h * u-z)^{2}\right)\right)=0.0004$ and $\operatorname{mean}(G *(h * u-z))=0.0003$. Figure 10.b displays the results obtained when $\sigma_{G}=10$. The values obtained are RMSE $=8.9145, \operatorname{mean}\left(G *(h * u-z)^{2}\right)=0.7967$, variance $\left.\left(G *(h * u-z)^{2}\right)\right)=0.00014$ and mean $(G *(h * u-z))=0.00007$. In this case, if we choose smaller values of $\sigma_{G}$, as $\sigma_{G}=3$, we are not able to eliminate all noise.

As it is recognized in [23], penalization methods permit to enforce the constraint very quickly but are ill-conditioned when the penalization parameter is large and we need many iterations to minimize total variation. In contrast, Uzawa's method is not as efficient as penalization to enforce the constraint but they provide a neat tool when the numerical accuracy in the constraint is not necessarily needed. This is in agreement with our experiments. The authors advocate for a final step of a few iterations based on the penalization method starting with the result obtained with Uzawa's method.

\section{Conclusions}

We have proposed a Total Variation based restoration model with local constraints. The inclusion of the image acquisition model as a single constraint expressing that the variance of the noise is bounded by $\sigma^{2}$ does not give satisfactory results if we wish to simultaneously recover textured regions and obtain a good denoising of the image. For that we have proposed to include the image acquisition model as a set of local constraints, one for each pixel of the image, expressing the fact that the variance of the noise can be estimated from the residuals $h * u-z$ if we use a neighborhood of each pixel. The minimization of the total variation subject to this family of constraints can be accomplished by means of Uzawa's algorithm. We have displayed some experiments showing that the use of local constraints permits to attain the desired objectives.

Acknowledgement. The first author was partially supported by Programa de Desarrollo Tecnológico in Uruguay (PDT S/C/OP/17/01). The second, third and fourth authors acknowledges partial support by PNPGC project, reference BFM2003-02125. The third author also acknowledges partial support by 

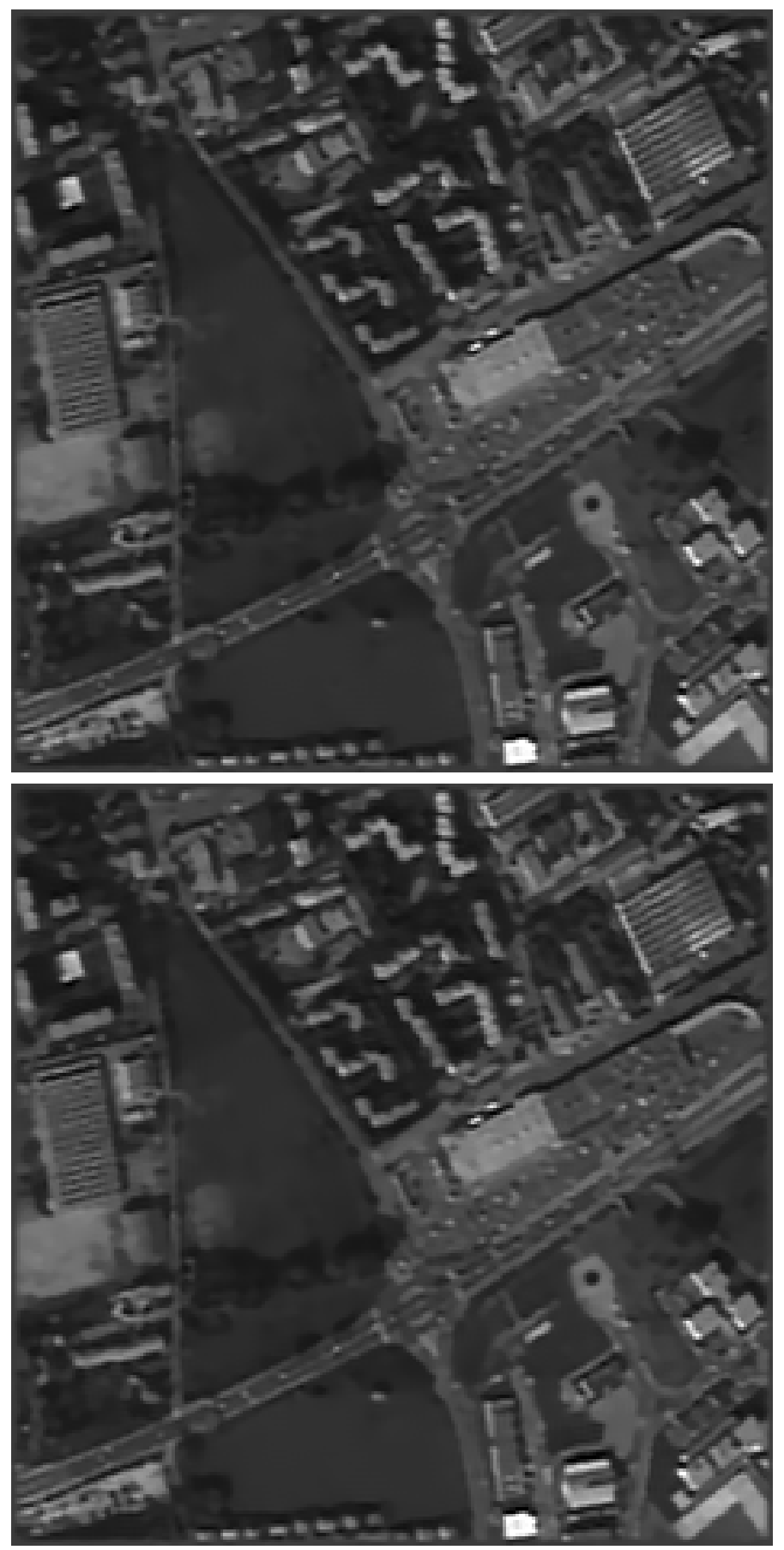

Figure 8: Restored image with local Lagrange multipliers and $\bar{\sigma}=1.1$. a) The top Figure displays the result obtained with $\beta=0, \bar{\sigma}=1.1$ and $\sigma_{G}=3$. We have $\operatorname{RMSE}=9.2279$, mean $\left.\left(G *(h * u-z)^{2}\right)\right)=$ 1.1237, variance $\left.\left(G *(h * u-z)^{2}\right)\right)=0.0073$. b) The bottom Figure displays the result obtained with $\beta=0, \bar{\sigma}=1.1$ and $\sigma_{G}=6.5$. We have $\left.\operatorname{RMSE}=9.3193, \operatorname{mean}\left(G *(h * u-z)^{2}\right)\right)=1.1378$, $\left.\operatorname{variance}\left(G *(h * u-z)^{2}\right)\right)=0.0050$. 

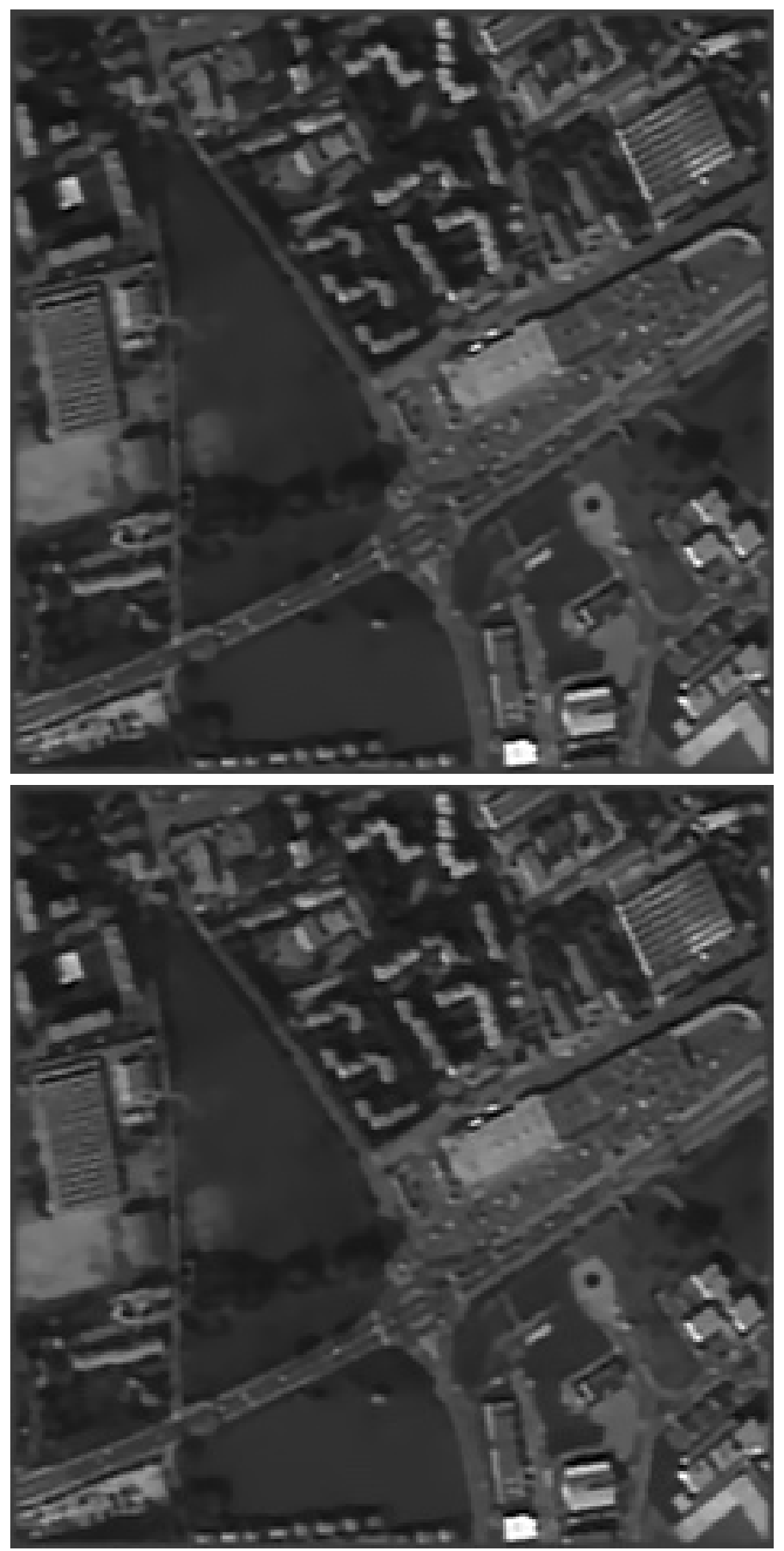

Figure 9: Restored image with local Lagrange multipliers and $\bar{\sigma}=1.2$. a) The top Figure displays the result obtained with $\beta=0, \bar{\sigma}=1.2$ and $\sigma_{G}=3$. We have RMSE $=9.4652$, mean $\left.\left(G *(h * u-z)^{2}\right)\right)=$ 1.3019, variance $\left.\left(G *(h * u-z)^{2}\right)\right)=0.0196$. b) The bottom Figure displays the result obtained with $\beta=0, \bar{\sigma}=1.2$ and $\sigma_{G}=6.5$. We have $\operatorname{RMSE}=9.5763$, mean $\left.\left(G *(h * u-z)^{2}\right)\right)=1.3228$, $\left.\operatorname{variance}\left(G *(h * u-z)^{2}\right)\right)=0.0163$. 

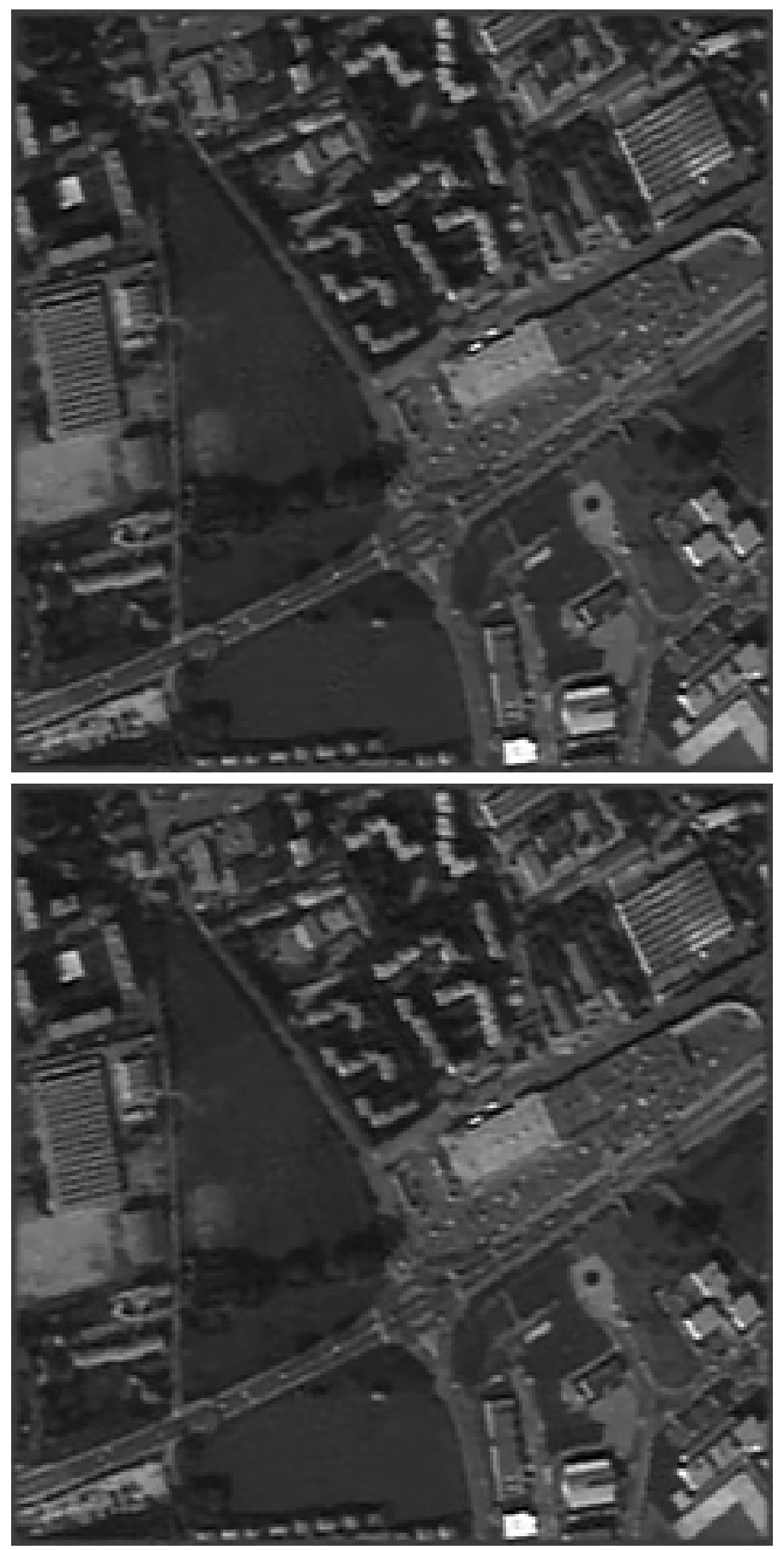

Figure 10: Restored image with local Lagrange multipliers and $\bar{\sigma}=0.9$. a) The top Figure displays the result obtained with $\beta=0, \bar{\sigma}=0.9$ and $\sigma_{G}=6.5$. We have $\left.\operatorname{RMSE}=8.9157, \operatorname{mean}\left(G *(h * u-z)^{2}\right)\right)=$ 0.7935 , variance $\left.\left(G *(h * u-z)^{2}\right)\right)=0.0004$. b) The bottom Figure displays the result obtained with $\beta=0, \bar{\sigma}=0.9$ and $\sigma_{G}=10$. We have RMSE $=8.9145$, mean $\left.\left(G *(h * u-z)^{2}\right)\right)=0.7967$, $\left.\operatorname{variance}\left(G *(h * u-z)^{2}\right)\right)=0.00014$. 
the Departament d'Universitats, Recerca i Societat de la Informació de la Generalitat de Catalunya. The fourth author acknowledges partial support by the Institute of Mathematics and its Applications (University of Minnesota).

\section{References}

[1] R. Acar and C.R. Vogel, Analysis of Total Variation Penalty Methods for Ill-Posed Problems, Inverse Problems, 10 (1994), pp. 1217-1229.

[2] A. Almansa. Echantillonnage, Interpolation et Dtction. Applications en Imagerie Satellitaire. PhD thesis, Ecole Normale Suprieure de Cachan, 94235 Cachan cedex, France, December 2002.

[3] A. Almansa, V. Caselles, G. Haro, and B. Rougé. Restoration and zoom of irregularly sampled, blurred and noisy images by accurate total variation minimization with local constraints. To appear in Multiscale Modeling and Simulation.

[4] L. Ambrosio, N. Fusco and D. Pallara, Functions of Bounded Variation and Free Discontinuity Problems. Oxford Mathematical Monographs, 2000.

[5] H.C. Andrews and B.R. Hunt, Digital Image Restoration. Tech. Englewood Cliffs, NJ, Prentice Hall, 1977.

[6] L. Blanc-Féraud, P. Charbonnier, G. Aubert and M. Barlaud, Nonlinear image processing: modeling and fast algorithm for regularization with edge detection. Proceedings of the International Conference on Image Processing 1995, pp. 474-477, 1995.

[7] A. Bermúdez and C. Moreno. Duality methods for solving variational inequalities. Comput. Math. Appl., 7(1):43-58, 1981.

[8] M. Bertalmío, V. Caselles, B. Rougé, and A. Solé. TV based image restoration with local constraints. Journal of Scientific Computing, 19(1-3):95-122, 2003.

[9] H. Brezis. Operateurs Maximaux Monotones. North Holland, Amsterdam, 1973.

[10] A. Chambolle. An algorithm for total variation minimization and applications. Journal of Mathematical Imaging and Vision 20, 89-97, 2004.

[11] A. Chambolle and P. L. Lions, Image Recovery via Total Variation Minimization and Related Problems. Numerische Mathematik 76:167-188, 1997.

[12] T. F. Chan, G.H. Golub and P. Mulet, A Nonlinear Primal-Dual Method for Total Variation Based Image Restoration. SIAM J. Sci. Computing 20(6), 1964-1977, 1999.

[13] G. Demoment. Image reconstruction and restoration: Overview of common estimation structures and problems. IEEE Trans. on Acoustics, Speech and Signal Proc., 37(12):2024-2036, 1989.

[14] D. Donoho and I.M. Johnstone, Ideal spatial adaptation by wavelet shrinkage. Biometrika, 81(3), (1994), pp. 425-455.

[15] S. Durand, F. Malgouyres and B. Rougé, Image Deblurring, Spectrum Interpolation and Application to Satellite Imaging. Mathematical Modelling and Numerical Analysis, 1999. 
[16] P. Faurre. Analyse numérique. Notes d'optimisation. École Polytechnique. Ed. Ellipses, 1988.

[17] N.P. Galatsanos and A.K. Katsaggelos, Methods for Choosing the Regularization Parameter and Estimating the Noise Variance in Image Restoration and Their Relation. IEEE TRansactions on Image Processing 1, 322-336, 1992.

[18] C. Gasquet and P. Witomski. Analyse de Fourier et Applications. Ed. Masson, 1990.

[19] D. Geman and G. Reynolds, Constrained Image Restoration and Recovery of Discontinuities. IEEE Trans. Pattern Anal. Machine Intell., 14 (1992), pp. 367-383.

[20] G. Gilboa, N. Sochen, and Y.Y Zeevi, Texture Preserving Variational Denoising Using an Adaptive Fidelity Term. Proceedings VLSM, 2003, Nice, France, pp. 137-144, 2003.

[21] A.K. Katsaggelos, J. Biemond, R.W. Schafer, and R.M. Merserau, A Regularized Iterative Image Restoration Algorithm. IEEE TRansactions on Image Processing 39, 914-929, 1991.

[22] G. Koepfler, C. Lopez, and J. M. Morel. A multiscale algorithm for image segmentation by variational method. SIAM Journal on Numerical Analysis, 31(1):282-299, 1994.

[23] S. Lintner and F. Malgouyres, Solving a variational image restoration model which involves $L^{\infty}$ constraints. Inverse Problems, 20:815-831, 2004.

[24] F. Malgouyres and F. Guichard. Edge direction preserving image zooming: a mathematical and numerical analysis. SIAM Journal on Numerical Analysis, 39(1):1-37, 2001.

[25] L. Moisan, Extrapolation de spectre et variation totale ponderée, Proceedings of GRETSI, 2001.

[26] R. Molina, A. Katsaggelos, and J. Mateos. Bayesian and Regularization Methods for Hyperparameter Estimation in Image Restoration. IEEE Transactions on Image Processing, 8 (1999), 231-246.

[27] D. Mumford and J. J. Shah. Optimal approximation by piecewise smooth functions and associated variational problems. Communications on Pure and Applied Mathematics, 42:577-684, 1989.

[28] V.K. Rohatgi, An Introduction to Probability Theory and Mathematical Statistics. Wiley, 1976.

[29] B. Rougé, Théorie de l'echantillonage et satellites d'observation de la terre. Analyse de Fourier et traitement d'images, Journées X-UPS 1998.

[30] L. Rudin and S. Osher, Total Variation based Image Restoration with Free Local Constraints. Proc. of the IEEE ICIP-94, vol. 1, Austin, TX, 1994, pp. 31-35.

[31] L. Rudin, S. Osher, and E. Fatemi. Nonlinear total variation based noise removal algorithms. Physica D, 60:259-268, 1992.

[32] D. Strong, P. Blomgren, and T. Chan. Spatially adaptative local feature driven total variation minimizing image restoration. Technical report, CAM Report, UCLA.

[33] A. N. Tikhonov and V. Y. Arsenin. Solutions of Ill-Posed Problems. V. H. Winston \& Sons, Washington, D.C.: John Wiley \& Sons, New York, 1977.

[34] L. Vese. A study in the BV space of a denoising-deblurring variational problem. Applied Mathematics and Optimization, 44:131-161, 2001. 
[35] C. R. Vogel and M. E. Oman, Iterative Methods for Total Variation Denoising, SIAM J. Sci. Computing, 17(1):227238, 1996. 